\title{
Topological blocking in quantum quench dynamics
}

\author{
G. Kells, ${ }^{1,2}$ D. Sen, ${ }^{3}$ J. K. Slingerland, ${ }^{1,4}$ and S. Vishveshwara ${ }^{5}$ \\ ${ }^{1}$ Department of Mathematical Physics, National University of Ireland, Maynooth, Ireland \\ ${ }^{2}$ Dahlem Center for Complex Quantum Systems and Fachbereich Physik, Freie Universität Berlin, Arnimallee 14, 14195 Berlin, Germany \\ ${ }^{3}$ Centre for High Energy Physics, Indian Institute of Science, Bangalore 560 012, India \\ ${ }^{4}$ Dublin Institute for Advanced Studies, School of Theoretical Physics, 10 Burlington Road, Dublin, Ireland \\ ${ }^{5}$ Department of Physics, University of Illinois at Urbana-Champaign, Urbana, Illinois 61801-3080, USA
}

(Received 17 January 2014; revised manuscript received 30 May 2014; published 26 June 2014)

\begin{abstract}
We study the nonequilibrium dynamics of quenching through a quantum critical point in topological systems, focusing on one of their defining features: ground-state degeneracies and associated topological sectors. We present the notion of "topological blocking," experienced by the dynamics due to a mismatch in degeneracies between two phases, and we argue that the dynamic evolution of the quench depends strongly on the topological sector being probed. We demonstrate this interplay between quench and topology in models stemming from two extensively studied systems, the transverse Ising chain and the Kitaev honeycomb model. Through nonlocal maps of each of these systems, we effectively study spinless fermionic $p$-wave paired topological superconductors. Confining the systems to ring and toroidal geometries, respectively, enables us to cleanly address degeneracies, subtle issues of fermion occupation and parity, and mismatches between topological sectors. We show that various features of the quench, which are related to Kibble-Zurek physics, are sensitive to the topological sector being probed, in particular, the overlap between the time-evolved initial ground state and an appropriate low-energy state of the final Hamiltonian. While most of our study is confined to translationally invariant systems, where momentum is a convenient quantum number, we briefly consider the effect of disorder and illustrate how this can influence the quench in a qualitatively different way depending on the topological sector considered.
\end{abstract}

DOI: 10.1103/PhysRevB.89.235130

PACS number(s): 71.10.Pm, 75.10.Jm, 03.65.Vf

\section{INTRODUCTION}

Over the past years, there has been a revival of interest in the topics of topological systems and nonequilibrium critical dynamics stemming from the latest advances exhibited in a variety of condensed-matter and cold atomic systems [1-23]. The synergy of the two topics, namely, quench dynamics in topological systems, is still in its infancy $[14,16,19,20,23]$ but promises to form a rich and complex avenue of study. While previous works have targeted the formation of edge states and bulk defects that are characteristic of topological systems, in this work we focus in particular on the role of ground-state degeneracy, another key characteristic of topological order.

Our work highlights special features of quenches that involve initializing a system in the ground state of a phase with a particular topological order and dynamically evolving this state through a topological phase transition; that is, the Hamiltonian is time dependent, and the ground states of the initial and final Hamiltonians have differing topological order. We consider topological aspects of systems having periodic boundary conditions, i.e., rings or tori, where the effect of degeneracies is clear-cut. This is different from open-bounded systems, where the dynamics can be complicated by edge effects, and from infinite systems, where topological aspects can often be completely hidden. Most dramatically, we find a phenomenon which we call topological blocking: due to a mismatch in degeneracies, some of the ground states of a topological system have no overlap with any of the ground states on the other side of the transition, regardless of how slowly the quench is performed.

We expect that our central observations apply to a wide range of topological systems. Our general setting involves two gapped phases with different degeneracies separated by a gapless critical point (or, more generally, a gapless region). Topological blocking is best seen by initializing the system in the phase with higher degeneracy. Over the evolution of the quench, as shown in Fig. 1, some of the topological sectors of this phase are forced to be lifted in energy as they pass through the gapless point so that no states in those sectors appear as ground states in the new phase. Nevertheless, the states in the original topological sectors may remain topologically distinct from each other, so they cannot be connected by the action of local operators. Hence, in a quantum quench between the phases, an initial state in a sector that has its energy lifted evolves within that sector. The time-evolved state after the quench thus has zero overlap with any of the ground states in the final phase.

It is worth noting that a similar situation can happen when the quench preserves a symmetry which is spontaneously broken in the phase transition (if the Hamiltonian is always symmetric and the system starts in a symmetric state, it will stay in a symmetric state even if the ground state after the transition is not symmetric). However, in reality this phenomenon would have to be artificially engineered because, typically, there will always be some minor symmetry-breaking perturbations present during and after the quench. In contrast, topologically distinct ground states have the property that they are not connected by local operators (in contrast to ground states with different symmetry); thus, this blocking behavior is expected to be more natural in topological systems.

The role of the topological sector, while directly obvious for topological blocking, is also apparent when considering 


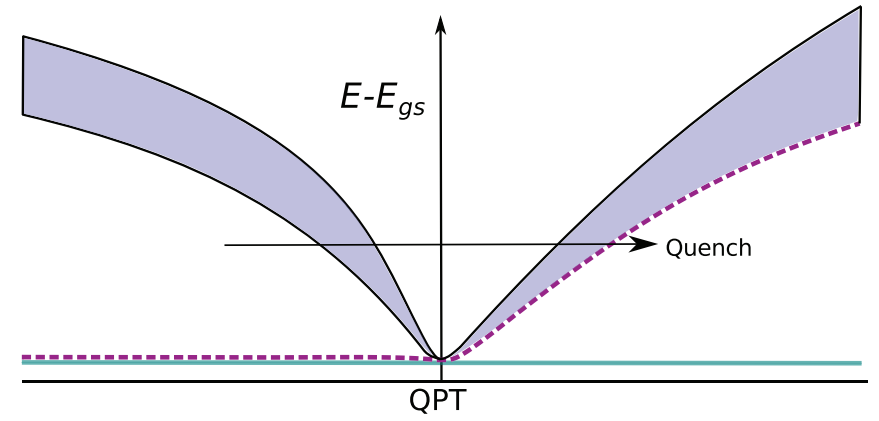

FIG. 1. (Color online) An example of topological blocking in which the quench goes from a doubly degenerate gapped phase to a nondegenerate gapped phase, as happens, for instance, in the one-dimensional spinless $p$-wave superconductor. Upon closing the gap at the critical point, one of the degenerate states in the initial phase is lifted into the continuum of excited states in the final phase. This time-evolved initial state has no overlap with the final ground state.

state evolution within the sector. We find that an effective indicator of sectoral dependence is the overlap of the timeevolved state with the lowest-energy state of the instantaneous quenched Hamiltonian within the same sector (sectoral ground state). Figure 2 shows an example illustrating such timedependent wave function overlaps for a quench from a doubly degenerate phase to a nondegenerate phase; the overlaps within the two sectors, labeled by parity, show a clear difference in their evolution during the quench, exhibiting the most pronounced features in the vicinity of the critical point. While the quantitative difference is obvious, under certain easily accessible circumstances, there can also be a qualitative difference if, unlike the absolute ground state, some

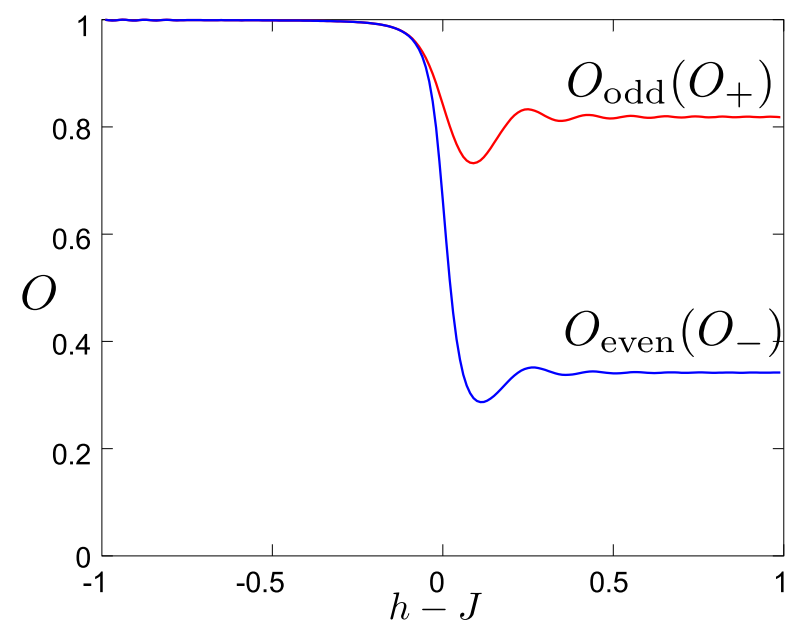

FIG. 2. (Color online) Typical quench data for the onedimensional spinless $p$-wave superconductor on an $N=60$ site ring show the overlap of the time-evolved ground state of the initial Hamiltonian in one topological sector with the lowest-energy state of the final Hamiltonian within that sector. (This is not always the global ground state of the final Hamiltonian.) As a result of the topological blocking, the odd-fermion sector with periodic boundary conditions has a higher final overlap than the even-fermion sector. See Sec. II for details. of the sectoral ground states in the postquench phase are not separated by a gap from the spectrum of excited states. It is worth mentioning here that these systems still respect the wellstudied Kibble-Zurek mechanism [2-5,7,8], which applies to systems with local as well as topological order and predicts power-law scaling as a function of quench rate in various quantities related to postquench excitations. The dependence on topological sectors rides above such scaling and, among the typical Kibble-Zurek quantities, such as residual energy or defect density, is most strongly manifest in wave-function overlaps.

The interplay between topology and quench dynamics provides new insights into each of these respective aspects. Our treatment shows that the quench dynamics between phases that have different ground-state degeneracies acts as a fine probe of topological order and examines some of its more subtle issues. For example, the notion of topological blocking highlights the fact that the number of topologically distinct subspaces (sectors) of the Hilbert space of a system may exceed the ground-state degeneracy; there may be topological sectors which are "hidden" at low energy but which nevertheless play a role in quantum quenches. In terms of quench physics, we bring attention to the concept that there typically exist multiple sectors in a system with topological order, which could show distinctly different dynamics. Understanding these quenches is also essential for the implementation of topologically fault-tolerant quantum computation schemes [24-26] where collective transitions between topological and nontopological phases (see, for example, Ref. [27]) represent a potential source of decoherence. The topological blocking mechanism and the fact that the hidden topological sectors need not be gapped (as we show below) present a further complication for such schemes.

In what follows, we perform an analysis of the features we discussed above within the context of two topological systems that can effectively be described as spinless fermionic $p$-wave paired superconductors. We first study the quantum Ising chain in a transverse magnetic field, perhaps one of the most celebrated systems in condensed matter for offering a tractable solution and rich physics, one with plentiful studies even in the context of quenching [7,8,28-31]. The second system, the Kitaev honeycomb model, is also special in its analytically soluble structure [32-39] and has also received significant attention in the context of quenching [9]. The transverse Ising model maps to a $p$-wave superconducting chain [40], while the honeycomb lattice model maps to a $p+i p$ superconductor coupled to a $\mathbb{Z}_{2}$ gauge field $[32,33,35,36]$, the latter thus being a natural two-dimensional extension of the former. In both models, we carefully pinpoint how topological blocking comes about, using the structure of the Bogoliubov-de Gennes (BdG) Hamiltonians, and perform a detailed analysis of the difference in postquench behavior for quenches within different topological sectors.

In the transverse Ising system, the topological sectors are identified in terms of fermion number parity, which is naturally accounted for in the boundary conditions of the ring. In this case, the quench involves going from a phase having a double degeneracy associated with even and odd parity to another phase having a unique ground state characterized by one of the two parities. It is important to note, however, that in 
the spin language, the phase with the two degenerate ground states corresponds to a ferromagnet that spontaneously breaks local Ising $\mathbb{Z}_{2}$ symmetry, while that with the unique ground state corresponds to a phase with spin polarization along the magnetic field.

Here we have hit upon an interesting phenomenon. The transverse Ising spin chain is not a topological model, while the corresponding fermionic model, whose Hamiltonian is unitarily equivalent, is. The reason that this can happen is that these two seemingly equivalent models have different local degrees of freedom (fermions versus spins) and hence different sets of local operators. This notion of locality plays a crucial role in determining whether a phase is topological or not. On this point note that for the superconducting model there is no natural local observable that will detect the parity of the total number of fermions. Furthermore, while it is true in this case that single-fermion creation and annihilation operators can connect the degenerate ground states, one assumes that (short of connecting the system to a particle bath) there is no process that can do this.

We will initially consider topological blocking in this onedimensional fermionic system because it provides a simple and familiar context which illustrates the concepts and because it is directly relevant to current and proposed experiments. In fact, the superconducting fermionic model, with open boundary conditions, is currently at the heart of considerable excitement about the possible detection of Majorana end states associated with the bulk topological order [41]. The corresponding Ising spin ring is also of interest for a number of reasons. First of all, the analysis for the superconducting ring system where parity is preserved is just as valid for idealized spin-quench scenarios where the local $\mathbb{Z}_{2}$ symmetry is not explicitly broken. It appears that this aspect of the transverse Ising model has not been discussed in detail before. Second, the duality between the spin and fermion pictures allows us to carry over intuition from the conventional symmetry-breaking spin model to the fermionic model. For example, we will see that the eigenvalues of the symmetry operators in the spin model are directly analogous to flux/boundary conditions in the fermionic representation. Finally, the spin model is in many ways analogous to the topologically ordered twodimensional Kitaev honeycomb spin model treated later in the paper.

In the honeycomb lattice model the relevant phases are an Abelian phase with the topological order of the toric code, which is fourfold degenerate, and a non-Abelian phase with Ising-type topological order and threefold degeneracy. In this system, in contrast to the transverse Ising model, all ground states have the same parity and belong to the vortex-free sector. In this case, local operations necessarily excite either vortices or fermions and thus cannot connect one ground state to another. In this model then, both sides of the transition are topological in both the spin and the fermionic representations.

An overview of the paper is as follows. Section II discusses the transverse Ising case in depth, starting with a brief introduction, followed by its superconductor description, a discussion of degeneracies and the quench protocol, an explanation of topological blocking in terms of parity arguments, and, finally, detailed studies of quench behavior for different topological sectors. Section III gives a similar treatment of the Kitaev honeycomb model. In Sec. IV, we perform initial studies of quenches in these systems in the presence of disorder as a means of demonstrating robustness against local perturbations as well as the marked difference in topological sectors in situations where the blocked sector can access a slew of low-lying excitations. We conclude with a short summary and outlook in Sec. V.

\section{THE TRANSVERSE ISING MODEL}

The transverse Ising model in one dimension is one of the best studied exactly solvable models (see Ref. [42] for a thorough treatment). As is commonly done to solve almost any aspect of the model, the nonlocal Jordan-Wigner transformation is used to map it to a beautiful prototype of a topological system: a spinless fermionic, one-dimensional $p$-wave superconductor. Here, after introducing the model, we reiterate the fermionization procedure, taking into account the subtleties associated with periodic boundary conditions and fermion parity. We carefully describe the link between fermion parity, topological degeneracy, the topological sectors on either side of the transitions, and their associated sectoral ground states. With these considerations in place, we show how topological blocking naturally comes about. We then study the dynamics of the quench in each topological sector, focusing on the overlap between the time-evolved initial ground state and instantaneous sectoral ground states. Our analytic treatment uses the Landau-Zener formalism typically applied of late to related quenches in homogeneous systems $[4,5,7,8]$, and we corroborate it with numerical studies.

The most frequently encountered form of the Hamiltonian for the transverse Ising model is given by

$$
H_{T I}=-J \sum_{\langle i j\rangle} \sigma_{i}^{x} \sigma_{j}^{x}-h \sum_{i} \sigma_{i}^{z} .
$$

Here, $\sigma^{i}$ denote spin-1/2 Pauli matrices, $J$ is an Ising ferromagnetic coupling, $h$ is a Zeeman magnetic field in the $z$ direction, and $\langle i j\rangle$ are nearest neighbors $i$ and $j$. (We set Planck's constant $\hbar=1$ throughout this paper.) If we take $J>0$ and $h>0$, the system has two phases, ferromagnetic and paramagnetic. The ordered Ising ferromagnet along the $x$ direction occurs for $h<J$, while the paramagnetic phase occurs for $h>J$. The two phases are separated by a quantum critical point at $h=J$.

The ground-state degeneracies of the two phases can be discerned by looking at the Hamiltonian in some simple limits. In the paramagnetic limit, $J=0$, we see that the ground state is simply the nondegenerate state fully polarized along the direction of the Zeeman magnetic term,

$$
|\mathrm{gs}\rangle=|\overline{0}\rangle=|00 \cdots 00\rangle
$$

where, for the spin state on a single site, $|0\rangle=[1,0]^{T}$ and $|1\rangle=$ $[0,1]^{T}$ in the eigenvalue basis of $\sigma^{z}$. The overbar denotes the quantum state for the entire collection of sites. In the opposite ferromagnetic limit, $h=0$, there are two degenerate ground 
states given by superpositions of

$$
|\overline{+}\rangle=|++\cdots++\rangle, \quad|\overline{-}\rangle=|--\cdots--\rangle,
$$

where $|+\rangle=[1,1]^{T} / \sqrt{2}$ and $|-\rangle=[1,-1]^{T} / \sqrt{2}$ are the eigenstates of $\sigma^{x}$. The system is symmetric under a global $\pi$ rotation around the $z$ axis, given (up to a global phase) by the string operator

$$
T_{z}=\prod_{i} \sigma_{i}^{z}
$$

This nonlocal operator maps the $|\overline{+}\rangle$ and $|\bar{\beth}\rangle$ states into each other, while $|\overline{0}\rangle$ is left invariant. After fermionization, $T_{z}$ is associated with fermion parity and topological degeneracy; note that $T_{z}$ is conserved even if the couplings in Eq. (1) are allowed to be functions of space.

\section{A. Fermionized topological superconductor and solution}

The original fermionic solution for the transverse Ising chain can be traced to Pfeuty [43], who used a transformation similar to Lieb et al. [44]. Indeed, the fermionic dispersion relation for the transverse Ising can be seen to be identical to that of the $X Y$ model solved by Lieb et al. Here too we employ their extensively used Jordan-Wigner transformations to define the position space fermionic excitations (see, for example, Refs. [7,8,29])

$$
c_{i}^{\dagger}=\left(\prod_{j<i} \sigma_{j}^{z}\right) \sigma_{i}^{-}, \quad c_{i}=\left(\prod_{j<i} \sigma_{j}^{z}\right) \sigma_{i}^{+} .
$$

The state $|\overline{0}\rangle$ given in Eq. (2) is therefore the fermionic vacuum state. At any site $i$, we have $\sigma_{i}^{z}=(-1)^{c_{i}^{\dagger} c_{i}}$. Hence $T_{z}$ gives the parity of the total fermion number,

$$
T_{z}=(-1)^{N_{F}}, \quad N_{F}=\sum_{i} c_{i}^{\dagger} c_{i} .
$$

In terms of fermion operators the Hamiltonian takes the superconducting form

$$
\begin{aligned}
H= & h \sum_{i=1}^{N}\left(2 c_{i}^{\dagger} c_{i}-1\right)-J \sum_{i=1}^{N-1}\left(c_{i}^{\dagger}-c_{i}\right)\left(c_{i+1}^{\dagger}+c_{i+1}\right) \\
& +J T_{z}\left(c_{N}^{\dagger}-c_{N}\right)\left(c_{1}^{\dagger}+c_{1}\right),
\end{aligned}
$$

where $N$ is the number of sites on the ring. This superconducting Hamiltonian for spinless fermions has an on-site chemical potential $\mu=-2 h$, nearest-neighbor hopping of strength $w=J$, and anomalous $p$-wave pairing terms also of strength $\Delta=J$. A generalization of this model having $w \neq \Delta$ can be obtained by considering an $X Y$ spin chain instead of an Ising spin chain [8]; the main results of this section also hold for this case.

The boundary conditions of the system are encoded in the operator $T_{z}$. To select the periodic sector we replace the operator $T_{z}$ with its eigenvalue -1 , corresponding to an odd number of fermions. To select the antiperiodic sector we replace the operator with the eigenvalue +1 , corresponding to even parity.
The Hamiltonian can be written in momentum space as a sum of BdG Hamiltonians,

$$
\begin{aligned}
H & =\sum_{0 \leqslant k \leqslant \pi}\left[\begin{array}{ll}
c_{k}^{\dagger} & c_{-k}
\end{array}\right] H_{k}\left[\begin{array}{c}
c_{k} \\
c_{-k}^{\dagger}
\end{array}\right], \\
H_{k} & =\left[\begin{array}{cc}
\xi_{k} & \Delta_{k} \\
\Delta_{k}^{*} & -\xi_{k}
\end{array}\right], \\
\xi_{k} & =-2 h-2 J \cos (k), \\
\Delta_{k} & =2 J \sin (k) .
\end{aligned}
$$

The BdG Hamiltonians $H_{k}$ can be diagonalized by a Bogoliubov transformation. Namely, we may write

$$
\begin{aligned}
H & =\sum_{0 \leqslant k \leqslant \pi} \epsilon_{k}\left(\gamma_{k}^{\dagger} \gamma_{k}+\gamma_{-k}^{\dagger} \gamma_{-k}-1\right), \\
\epsilon_{k} & =\sqrt{\xi_{k}^{2}+\left|\Delta_{k}\right|^{2}},
\end{aligned}
$$

in terms of the Bogoliubov-Valatin operators

$$
\begin{aligned}
\gamma_{k} & =u_{k} c_{k}-v_{k} c_{-k}^{\dagger}, & \gamma_{k}^{\dagger} & =u_{k}^{*} c_{k}^{\dagger}-v_{k}^{*} c_{-k}, \\
\gamma_{-k} & =u_{k} c_{-k}+v_{k} c_{k}^{\dagger}, & \gamma_{-k}^{\dagger} & =u_{k}^{*} c_{-k}^{\dagger}+v_{k}^{*} c_{k},
\end{aligned}
$$

with

$$
\begin{aligned}
& u_{k}=\sqrt{\left(1+\xi_{k} / \epsilon_{k}\right) / 2}, \\
& v_{k}=-\sqrt{\left(1-\xi_{k} / \epsilon_{k}\right) / 2} .
\end{aligned}
$$

(We will see below that the modes with $k=0$ and $\pi$ require a special analysis since they satisfy $k=-k$. Further, $\Delta_{k}=0$ for these modes; hence, $\varepsilon_{k}=\left|\xi_{k}\right|$.) We see that in both phases of the model, the excitation energy $\epsilon_{k}$ is gapped for all $k$; the minimum energy lies at $k=0$ with $\epsilon_{0}=2|h-J|$. At the critical point $h=J$, the system is gapless and $\epsilon_{k}=0$ for $k=0$.

With regard to the topological aspects of the superconductor, the ferromagnetic phase, having a double ground-state degeneracy, maps to a topological phase, and the nondegenerate paramagnetic phase maps to a topologically trivial phase. This can be seen from standard Berry's phase analyses of the momentum eigenstate spinor structure [40,45]. Alternatively, it is common to consider the Kitaev chain, a finite open-chain version of the Hamiltonian in Eq. (7), which naturally lacks the $T_{z}$ term associated with the (anti)periodic boundary conditions of the ring geometry. The topological phase then has free Majorana modes at each end which lie at zero energy if the chain length is much larger than the decay length of these end modes. The Majorana end modes together form a Dirac fermion state which can either be occupied or unoccupied, thus accounting for the double degeneracy and fermion parity. By direct analogy with the paramagnetic regime we see that for open systems the ground state in the topologically trivial phase is characterized by the lowest fermion occupancy (all spins pointing along the magnetic field) and thus always has even fermion parity. It is also of interest to note that the boundary conditions on the fermionic ring, which in our analysis emerged from the spin model as eigenvalues of $T_{z}$, can be given a natural physical interpretation by allowing for scenarios where the ring may be threaded by a $\pi$ flux. 
As we will see in the next section, the relationship between topological degeneracy and fermion parity is more subtle in the ring geometry than for the open chain and requires some careful consideration. The following analysis will also form a natural template for the subsequent examination of the Kitaev honeycomb on a torus, which can, in a number of ways, be viewed as a two-dimensional extension of the transverse Ising system.

\section{B. Topological degeneracy}

We now describe the ground states of the model in terms of the occupation numbers of the fermionic modes and explain in detail how the topological sectors of the Ising chain are connected to fermion parity. In particular, we show that there is always a ground state of the system with an even fermion number, while a ground state with an odd fermion number exists only in the ferromagnetic phase. In the paramagnetic phase, the lowest-energy state with an odd fermion number is part of a band which is gapped away from the true (even fermion number) ground state. A schematic of the spectrum of the model highlighting these features is shown in Fig. 3.

We focus first on the case where the number of sites $N$ is even. In the even-fermion antiperiodic sector, the allowed momenta are then given by $k=\frac{2 \pi}{N}\left(n+\frac{1}{2}\right)$ with integer $n \in$ $[-N / 2, N / 2-1]$. Crucially, note that the values of $k$ do not

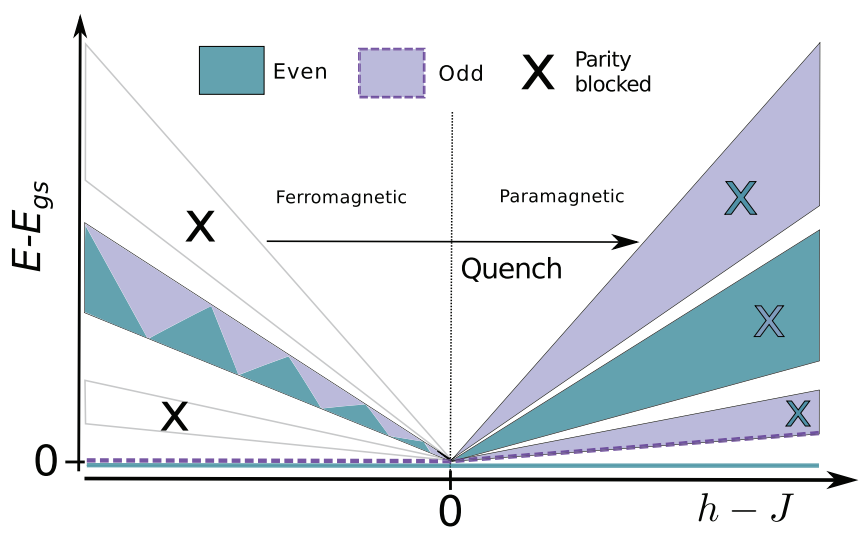

FIG. 3. (Color online) Schematic of the spectrum of the transverse Ising ring as a function of $h-J$. In the ferromagnetic phase, the ground state is doubly degenerate in the thermodynamic limit, and the excitation spectrum consists of bands of states with both even and odd fermion numbers. These states are created from the two ground states using pairs of $\gamma^{\dagger}$ operators. In particular, there are no energy levels with an odd number of $\gamma^{\dagger}$ excitations over one of the ground states. We explicitly indicate these levels as the parity-blocked regions. In the paramagnetic phase there is a unique ground state with an even number of fermions. The lowest excited band consists of odd-fermion-number states which are, however, not created by single $\gamma^{\dagger}$ from the ground state. Further bands are created from ground state and the lowest band using pairs of $\gamma^{\dagger}$ operators. The purple dashed line indicates that, in the adiabatic limit, the odd sectoral ground state of the ferromagnetic phase flows to the lowest-energy state in the paramagnetic phase. include 0 and $\pi$. The ground state is given by

$$
|\mathrm{gs}\rangle_{\text {even }}=\prod_{0<k<\pi, \frac{N k}{\pi} \text { odd }}\left(u_{k}+v_{k} c_{k}^{\dagger} c_{-k}^{\dagger}\right)|\overline{0}\rangle,
$$

where $k$ spans the restricted set of momenta described above. The energy of this state is given by $E_{\mathrm{gs}}=-\frac{1}{2} \sum \epsilon_{k}$, where the sum runs over all allowed momenta.

In the odd-fermion periodic sector the allowed momenta are given by $k=\frac{2 \pi n}{N}$ with integer $n \in[-N / 2, N / 2-1]$. These include the momenta $k=0, \pi$, which need to be treated carefully. In the ferromagnetic phase occurring for $J>h \geqslant 0$, we have $u_{0}=0, v_{0}=1$; hence $\gamma_{0}=c_{0}^{\dagger}$. From Eq. (9) we see that the contribution of this mode to the Hamiltonian is then just $H_{0}=2(h-J)\left(c_{0}^{\dagger} c_{0}-1 / 2\right)$, and thus the fermionic state with the $k=0$ mode occupied has lower energy compared to that with the mode unoccupied. We also have $u_{\pi}=1, v_{\pi}=0$, so that $\gamma_{\pi}=c_{\pi}$, and similar arguments show that the energetically favorable state has the $k=\pi$ mode unoccupied. Hence the ground state is given by

$$
|\mathrm{gs}\rangle_{\text {odd }}=c_{0}^{\dagger} \prod_{0<k<\pi, \frac{N k}{\pi} \text { even }}\left(u_{k}+v_{k} c_{k}^{\dagger} c_{-k}^{\dagger}\right)|\overline{0}\rangle .
$$

As this state is annihilated by all the $\gamma_{k}$ it has an energy given by $E_{\mathrm{gs}}=-\frac{1}{2} \sum \epsilon_{k}$, where the sum runs over all allowed momenta. In this phase, the values of $k$ become arbitrarily close to those of the even-fermion sector, and for $N \gg 1$ we get a twofold degenerate ground state.

The situation is quite different in the paramagnetic phase, which occurs for $h>J \geqslant 0$. The odd-fermion parity sector has a state with $k=0$ with $u_{0}=1, v_{0}=0$, so that $\gamma_{0}=c_{0}$. In principle, having the fermionic $k=0$ and $k=\pi$ modes unoccupied would be the lower-energy state. However, this would violate the odd parity of the sector. Given that as a function of $k, \epsilon_{k}$ has the smallest value for $k=0$, the state defined in Eq. (13) still does the best in terms of minimizing the energy within the odd sector. In this case, $c_{0}^{\dagger}=\gamma_{0}^{\dagger}$, so we are looking at the state in Eq. (12) with an extra $\gamma_{0}^{\dagger}$ excitation. This state is the lowest state of a band which can be obtained by exciting the system at nonzero momentum using $\gamma_{k}^{\dagger}$ instead of $\gamma_{0}^{\dagger}$. Thus, Eq. (13) corresponds to the sectoral ground state in the paramagnetic phase. However, the state now possesses energy $E_{\mathrm{gs}}=\epsilon_{0}-\frac{1}{2} \sum_{k} \epsilon_{k}$. In the limit $N \gg 1$, we see that the ground state in the odd-fermion sector lies at an energy which is higher than the ground state in the even-fermion parity sector by a finite amount equal to $\epsilon_{0}=2(h-J)$.

Now let us briefly discuss what happens if $N$ is odd. Then in the even-fermion antiperiodic sector, the allowed momenta are given by $k=\frac{2 \pi}{N}\left(n+\frac{1}{2}\right)$ with integer $n \in[-(N-1) / 2,(N-$ 1)/2], which includes the $k=\pi$ term but not $k=0$. In both the ferromagnetic and paramagnetic phases, the even sectoral ground state is still given by Eq. (12) (with the appropriate momentum quantization), and this state continues to be the absolute ground state. In the odd-fermion periodic sector, the allowed momenta are given by $k=\frac{2 \pi n}{N}$ with integer $n \in[-(N-1) / 2,(N-1) / 2]$, which includes the $k=0$ term but not $k=\pi$. Here, too, Eq. (13) remains the odd sectoral ground state and is another absolute ground state in the ferromagnetic phase but has higher energy in the paramagnetic 
phase. The situation is therefore similar in many ways to the case where $N$ is even.

To summarize, in the thermodynamic limit $N \gg 1$, the ground state of the system in the ferromagnetic phase has a double degeneracy, with one ground state lying in each of the sectors (even and odd fermion). In the paramagnetic phase, there is a unique ground state which lies in the even-fermion sector. The sectoral ground state in the odd-fermion sector lies in a band which is separated by a finite gap from the ground state in the even-fermion sector. To get an intuitive picture of how the degeneracy arguments derived from parity considerations connect with the spin picture described earlier, we can analyze the limit $h=0$. In this case the two ground states are given by

$$
\begin{aligned}
& |\mathrm{gs}\rangle_{\text {even }}=(|\bar{\mp}\rangle+|\overline{-}\rangle) / \sqrt{2}, \\
& |\mathrm{gs}\rangle_{\text {odd }}=(|\overline{+}\rangle-|\overline{-}\rangle) / \sqrt{2},
\end{aligned}
$$

where $|\overline{+}\rangle$ and $|\overline{-}\rangle$ are given in Eq. (3). These expressions serve to illustrate the important caveat that in the spin picture a local operator, such as $\sigma_{i}^{x}$, will connect these two ground states: $\sigma_{i}^{x}|\mathrm{gs}\rangle_{\text {even }}=|\mathrm{gs}\rangle_{\text {odd }}$. Indeed, this is true of any paritybreaking term. However, when one assumes a mean-field superconducting system of the form (7), it is implied that there are no single fermionic operators in play. We discuss this point further in Sec. IV.

\section{Quenching dynamics}

We now turn to the quench dynamics caused by slowly varying the transverse field in time, starting at $t=0$ at $h_{i}=0$ in the ground state of the ferromagnetic phase and ending at $t=T$ at $h_{f}=2 \mathrm{~J}$ in the paramagnetic phase. Note that the time evolution does not mix the even- and odd-fermion sectors; hence we will consider the time evolution in the two sectors separately.

Quench protocol. We consider a linear time dependence of the form

$$
h(t)=2 J t / T, \quad 0<t<T .
$$

By a slow variation, we mean that the dimensionless quantity $J T \gg 1$. Our analysis of quench dynamics partially follows those extensively performed in the context of Kibble-Zurek physics $[4,5,7,8]$ with the crucial difference that we explicitly consider fermion parity and momentum quantization associated with the topological sectors.

For any given set of $k$ modes (except 0 and $\pi$ ), the quench couples the two states in the occupation number basis $\left|n_{k}, n_{-k}\right\rangle=|0,0\rangle$ and $|1,1\rangle$. In this basis, the relevant dynamics is governed by the Hamiltonian

$$
H_{k}(t)=J\left(\begin{array}{cc}
\left(t-a_{k}\right) / \tau & b_{k} \\
b_{k} & -\left(t-a_{k}\right) / \tau
\end{array}\right),
$$

where Eqs. (8) imply that

$$
\tau=\frac{T}{4}, \quad a_{k}=\frac{T}{2} \cos (k), \quad b_{k}=2 \sin (k) .
$$

The instantaneous eigenvalues of the Hamiltonian in Eq. (15) have a minimum difference gap of $2 b_{k}$ at $t=a_{k}$. In our problem, the value of $a_{k}$ depends on $k$. Further, the initial and final values of $t-a_{k}$ are given by

$$
t_{i, k}=-\frac{T}{2} \cos (k), \quad t_{f, k}=T-\frac{T}{2} \cos (k),
$$

which also depend on $k$.

For each value of $k$, we study the quenching dynamics numerically as follows. We first calculate the quantities $u_{k}(t)$ and $v_{k}(t)$ in Eqs. (10) and (11) at the initial time $t=0$ with the initial value $h=h_{i}$. We then compute the time-ordered evolution operator

$$
U_{k}(t, 0)=\mathcal{T}\left\{\exp \left[-i \int_{0}^{t} H_{k}\left(t^{\prime}\right) d t^{\prime}\right]\right\}
$$

by dividing the time $t$ into $N_{t}$ steps of size $\Delta_{t}$ each (with $N_{t} \Delta_{t}=t$ ) and calculating

$$
U_{k}(t, 0) \approx \prod_{n=1}^{N_{t}}\left\{\exp \left[-i H_{k}\left(t_{n}\right) \Delta_{t}\right]\right\}
$$

where $t_{n}=(n-1 / 2) \Delta_{t}$. We then calculate

$$
\left(\begin{array}{c}
u_{k}^{*}(t) \\
-v_{k}^{*}(t)
\end{array}\right)=U_{k}(t, 0)\left(\begin{array}{c}
u_{k}^{*}(0) \\
-v_{k}^{*}(0)
\end{array}\right) .
$$

Finally, we compute the ground-state overlap by using the Onishi formula [46], which, for our $2 \times 2$ matrices, amounts to

$$
\begin{aligned}
O_{ \pm}(t) & =|\langle\mathrm{gs} \mid \psi(t)\rangle|^{2} \\
& =\prod_{k}\left|\left\langle\mathrm{gs}_{k} \mid \psi_{k}(t)\right\rangle\right|^{2} \\
& =\prod_{k}\left|u_{k}^{*}(t) u_{k}+v_{k}^{*}(t) v_{k}\right|,
\end{aligned}
$$

where the time-independent quantities $v_{k}$ and $u_{k}$ are those given in Eq. (11) and encode the instantaneous ground state. Here, the subscript \pm indicates the fermion parity and, consequently, the boundary conditions. The product over $k$ runs over the entire Brillouin zone from $-\pi$ to $\pi$ and, as discussed in previous sections, is restricted to certain values that depend on fermion parity. For a given momentum pair, the probability of being in the excited state of the Hamiltonian $H_{k}$ is

$$
p_{k}(t)=1-\left|\left\langle\mathrm{gs}_{k} \mid \psi_{k}(t)\right\rangle\right|^{2} .
$$

This excitation probability governs much of the postquench behavior. A plot of $1-p_{k}$ for a number of $k$ values can be seen in Fig. 4.

Analysis. Because the fermion number parity is conserved throughout the quench, we observe the topological blocking behavior described in the Introduction. Initializing the system in the ground state of the ferromagnetic/topological phase in the odd-parity sector, we observe that, even at adiabatically slow quench rates, this state does not evolve to the overall ground state (which has an even fermion number), but rather to the sectoral ground state in the odd-fermion-number band.

At nonadiabatic quench rates, we therefore consider the overlap of the time-evolved state with the sectoral ground state of the final Hamiltonian. Figure 2 shows a representative case for the overlap as a function of time for the odd- and even-fermion sectors; the two curves are clearly different. We 


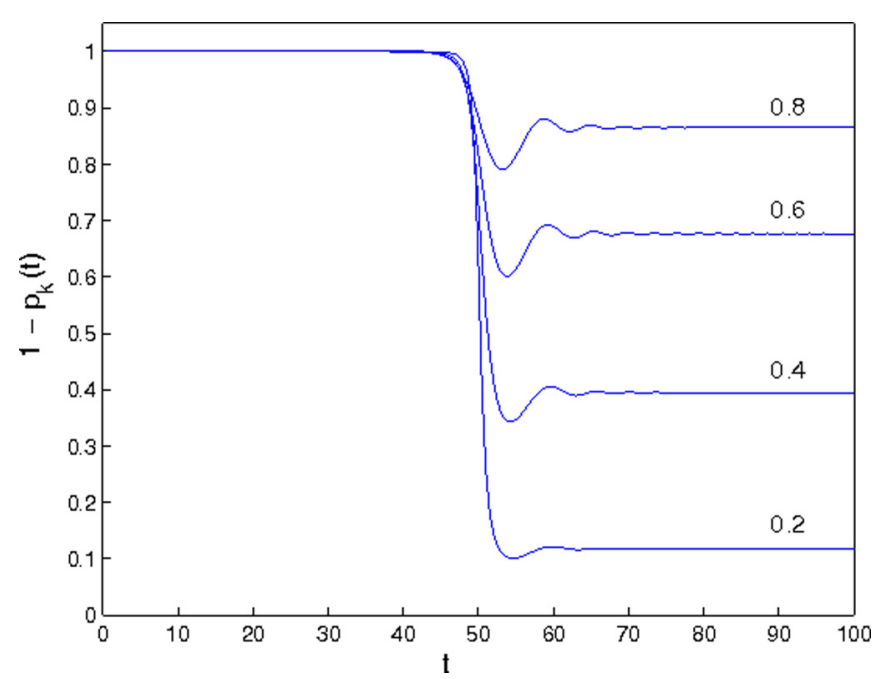

FIG. 4. (Color online) Overlap vs time for four two-level systems corresponding to $k \sqrt{J T}=0.2,0.4,0.6$. and 0.8 , with $h(t)=2 J t / T$ and $J T=100$. We have set $J=1$.

now analyze the detailed behavior of the time-evolved states, focusing on the contributions of each of the momentum modes and on the differences between sectors.

To begin with, we consider a simple problem in which the time $t$ in Eq. (15) goes from $-\infty$ to $\infty$, so that the value of $a_{k}$ is irrelevant. If we start in the ground state of $H(t)$ at $t=-\infty$, the probability of ending in the excited state of $H(t)$ at $t=\infty$ is given by the Landau-Zener expression [47-49]

$$
p_{k}(t=\infty)=e^{-\pi J b_{k}^{2} \tau}=e^{-\pi J T \sin ^{2}(k)} .
$$

This expression gives the correct limits $p_{k}(\infty) \rightarrow 0$ and 1 in the adiabatic $(T \rightarrow \infty)$ and sudden $(T \rightarrow 0)$ limits, respectively. Note that the momenta $k=0$ and $\pi$ are special; $b_{k}=0$ for these modes, and therefore $p_{k}=1$ for any quenching time $T$. Namely, these states do not change at all under quenching, and they change abruptly from the ground state to the excited state when $t$ crosses zero.

In the limit $J T \gg 1$, Eq. (23) shows that the excitation probability $p_{k}(\infty)$ is equal to 1 for $k=0$ and $\pi$ and becomes negligible when $k$ deviates from those points by an amount which is much larger than $1 / \sqrt{\pi J T}$. However, for our quench protocol, we see from Eq. (17) that the initial and final times, $t-a_{k}$, are functions of $k$; the time $t-a_{k}=0$ at which the two eigenvalues of the Hamiltonian are separated by the smallest amount $\left(2 b_{k}\right)$ is crossed only if $t_{i, k}<0$ and $t_{f, k}>0$, i.e., if $0 \leqslant k \leqslant \pi / 2$. Hence, the excitation probability is dominated only by the region near $k=0$; for exactly $k=0$, the two-level system undergoes a level crossing and $p_{0}=1$. The modes near $k=\pi$ never reach the minimum gap region, and for exactly $k=\pi$, the two-level system remains in the ground state with $p_{\pi}=0$. The behavior of the Landau-Zener transition exhibited by sets of $k$ modes and the evolution of the special $k=0$ mode is shown in Fig. 5.

In the adiabatic limit, we see that in the even-fermion sector, if we start in the ground state given in Eq. (12) at $h_{i}=0$, we reach the ground state in Eq. (12) at $h_{f}=2$. However, in the odd-fermion sector, if we start in the ground state in Eq. (13) at $h_{i}=0$, we reach the state in Eq. (13) at
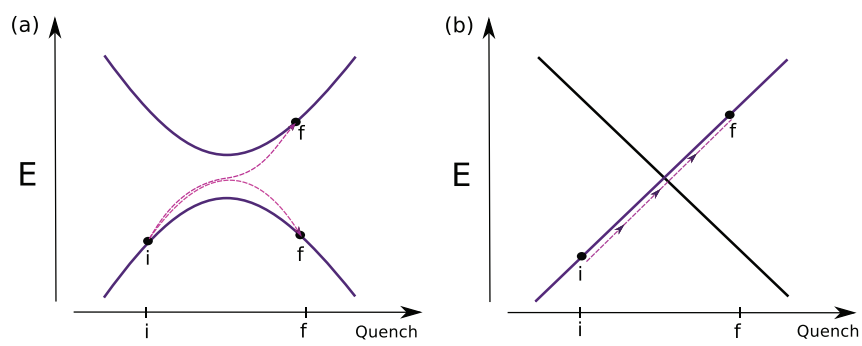

FIG. 5. (Color online) (a) Typical shift of probability amplitude in a Landau-Zener transition for states associated with generic $(k,-$ $k$ ) pairs. (b) The level crossing for the occupied $k=0$ state in the odd-fermion sector is at the heart of topological blocking; lack of coupling with the unoccupied mode and parity constraints force the $k=0$ state to go into the postquench excited state.

$h_{f}=0$ which is the ground state in that sector but which, as discussed above, is separated from the ground state of the final Hamiltonian by a finite gap. (Note that in the odd-fermion sector, the state with momentum $k=0$ does not change with time since the off-diagonal matrix element $b_{0}=0$ makes it impossible to have a transition between the two eigenstates of the Hamiltonian). Hence, an adiabatic time evolution takes a system from the initial ground state to the ground state of the final Hamiltonian in certain sectors but not in others, with the different sectors being distinguished from each other by a topological quantity, namely, the fermion parity in our model. This explicitly demonstrates topological blocking in this system.

Overlap at the final time. At $t=T$, the overlap between the final state reached and the actual ground state in a particular sector is given by

$$
\mathcal{O}(T)=\prod_{0<k<\pi}\left[1-p_{k}(T)\right] .
$$

In the limit $J T \gg 1$, we know that $p_{k}(T)$ is significant only for a range of $k$ of the order of $1 / \sqrt{\pi J T}$ near $k=0$. Let us consider the thermodynamic limit $N \gg 1$ and define a dimensionless scaling variable

$$
\bar{T}=\frac{\pi^{2} J T}{N^{2}} .
$$

Using the fact that the momenta in the even- and odd-fermion sectors are given by $(2 n+1) \pi / N$ and $(2 n+2) \pi / N$, where $n=0,1, \ldots, N / 2-1$, we can express the overlaps in the even- and odd-fermion sectors as

$$
\begin{aligned}
& \mathcal{O}_{\text {even }}(T) \approx \prod_{n=0}^{\infty}\left(1-e^{-\pi(2 n+1)^{2} \bar{T}}\right), \\
& \mathcal{O}_{\text {odd }}(T) \approx \prod_{n=0}^{\infty}\left(1-e^{-\pi(2 n+2)^{2} \bar{T}}\right),
\end{aligned}
$$

where we have made the approximation $\sin (k) \approx k$ in Eq. (23) since only the low-lying $k$ modes contribute a significant excitation probability. For the same reason we have changed the upper limit from $n=N / 2-1$ to $\infty$ since the overlap $1-p_{k}(T)$ rapidly approaches 1 once $n / N$ becomes a number of order, say, 0.1 , under the assumption $J T \gg 1$. 
A factor-by-factor comparison of the two expressions in Eqs. (26) shows that $\mathcal{O}_{\text {odd }}$ is larger than $\mathcal{O}_{\text {even }}$ for any value of $\bar{T}$. We therefore have the interesting result that the overlap between the final state and the sectoral ground state is higher in the odd-fermion sector than in the even-fermion sector, even though the final state in the odd-fermion sector has zero overlap with the ground state of the final Hamiltonian.

We can write the logarithms of the overlaps in Eqs. (26) as sums over $n$. In the limit $\bar{T} \rightarrow 0$, i.e., for $1 \ll J T \ll N^{2}$, the sums can be approximated by integrals. Ignoring the difference between $2 n+1$ and $2 n+2$ in Eqs. (26), which amounts to ignoring some subleading terms, we find that in both evenand odd-fermion sectors,

$$
\begin{aligned}
\log \mathcal{O}(T) & =\int_{0}^{\infty} d n \log \left(1-e^{-4 \pi n^{2} \bar{T}}\right) \\
& \approx-\frac{0.653}{\sqrt{\bar{T}}},
\end{aligned}
$$

and thus in the thermodynamic limit,

$$
\frac{\log \mathcal{O}(T)}{N}=-\frac{0.653}{\pi \sqrt{J T}} \text {. }
$$

Overlap at intermediate times. We now look at the overlap between the state reached at a finite time $t$ and the ground state at that time. This is given by the expression

$$
\mathcal{O}(t)=\prod_{0<k<\pi}\left[1-p_{k}(t)\right] .
$$

As has been analyzed in the context of Landau-Zener transitions [47-49], the analytic form of $p_{k}(t)$ can be expressed in terms of Weber functions. Numerically, we find that for a certain range of values of $\bar{T}$, the overlap $\mathcal{O}(t)$ of the system shows pronounced oscillations around $t=T / 2$ [i.e., when $h(t)=2 J t / T$ is going through the critical value of $J]$ before settling down at $t=T$ at a value which is around 0.5 , i.e., not very close to either 0 or 1 . We can estimate this range of values of $\bar{T}$ by looking at the overlap $1-p_{k}(t)$ as a function of time $t$ for some individual values of the momentum $k$. Assuming that $J T \gg 1$, we find the following. For $k \sqrt{J T} \ll 1$ (but not equal to 0 ), we have an almost sudden process. Hence the overlap stays close to 1 until we get close to $t=T / 2$, and then it rapidly changes to a very small value. Clearly, this would make the overlap of the system (which is a product of the overlaps for all values of $k$ ) very small. On the other hand, for $k \sqrt{J T} \gg 1$, we have an almost adiabatic process, and the overlap stays close to 1 at all times; such values of $k$ therefore make very little difference to the overlap of the system. Only if $k \sqrt{J T} \approx 0.47$ do we get a final overlap which is around 0.5. [This is consistent with Eq. (23) since $e^{-\pi(0.47)^{2}} \approx 0.5$.] These different kinds of behavior are shown in Fig. 4 for $J T=100$ and $k \sqrt{J T}=0.2$, $0.4,0.6$, and 0.8 . Thus, the behavior of the overlap of system that we are looking for, namely, oscillations near the critical point before settling down to a value around 0.5 , only occurs if the smallest nonzero value of $k$ satisfies $k \sqrt{J T} \approx 0.47$. Then this value of $k$ makes the dominant contribution to the overlap of the system at all times since all the higher values of $k$ contribute factors close to 1 to the overlap. Since the smallest nonzero value of $k=m \pi / N$, where $m=1$ and 2 in the even- and odd-fermion sectors, respectively, the value of
$\bar{T}$ where the final overlap of the system is around 0.5 is about $(0.47)^{2} \approx 0.22$ and $0.22 / 4=0.055$ for even- and odd-fermion sectors, respectively.

Figure 4 shows oscillations in the overlap near the critical region $t=T / 2$, which is equal to 50 for our choice of parameters. We can understand this by mapping the time evolution with the Hamiltonian in Eq. (15) to the Schrödinger equation of a particle moving in an inverted harmonic potential $[47,49]$. If we define the upper and lower components of the two-component wave function associated with the state $\left|\psi_{k}(t, 0)\right\rangle$ by $\psi_{1 k}$ and $\psi_{2 k}$, we can eliminate, say, $\psi_{2 k}$ to obtain the equation

$$
-\frac{d^{2} \psi_{1 k}}{d t^{2}}-\left(\frac{4 J\left(t-a_{k}\right)}{T}\right)^{2} \psi_{1 k}-i \frac{4 J}{T} \psi_{1 k}-J^{2} b_{k}^{2} \psi_{1 k}=0 .
$$

Since we are interested in the behavior of the solution of Eq. (30) when $J T k^{2} \approx 0.22$ is small (and $a_{k} \simeq T / 2$ ), we will ignore the last term, $J^{2} b_{k}^{2}=4 J^{2} \sin ^{2}(k)$, as this is much smaller than the other terms, such as $4 J / T$. The dominant behavior of the solutions of Eq. (30) is then given by $e^{-i 2 J(t-T / 2)^{2} / T}$. This explains the oscillations around $t=T / 2$. Further, as $t-T / 2$ moves away from zero, $e^{-i 2 J(t-T / 2)^{2} / T}$ oscillates more and more rapidly; this is qualitatively confirmed by the plots in Fig. 4. More details on the nature of the Landau-Zener oscillations can be found in Ref. [50].

To summarize the discussion in the last two paragraphs, the overlap in Eq. (29), in general, either stays close to 1 at all times or drops rapidly from 1 to 0 when the system crosses the quantum critical point at $t=T / 2$. The intermediate behavior in which the overlap drops to a value which is about halfway between 0 and 1 when $t$ crosses $T / 2$ occurs only when Eq. (29) is dominated by the smallest nonzero value of $k$, and that value of $k$ happens to satisfy $k \sqrt{J T} \approx 0.47$. For a system of size $N$, the smallest nonzero value of $k$ is given by $\pi / N$ and $2 \pi / N$ in the even- and odd-fermion sectors, respectively; from this we can deduce the value of $\sqrt{J T} / N$ at which the intermediate behavior occurs in the two sectors. When considered together, the highly sensitive nature of this quench behavior on the actual value of momentum, the dominance of a single mode in the net overlap, and the slightly different momentum quantization conditions for the two sectors together explain the markedly different quantitative behavior shown by the overlap in the two sectors in Fig. 2.

\section{Other quantities}

We have found the wave-function overlap plotted in Fig. 2 to be the most sensitive yet direct measure of the dependence of quench dynamics on topological sectors. In this context, we briefly discuss here other quantities that are commonly studied in quench dynamics and related Kibble-Zurek physics [2-5,7-12]. In fact, the behavior of several quantities can be traced back to that of the probability of excitation within each set of momentum modes, namely, that of $p_{k}(t)$, which was first introduced in Eq. (22).

Defect density. The well-studied Kibble-Zurek defect density is the cumulative sum of the excitation probabilities for all the modes, i.e., $n_{D} \sim \int d k p_{k}$. In terms of Ising spins, the 
defect density is a measure of how many spins are pointing in the energetically unfavorable direction in the final phase. In the final state reached at $t=\infty$, the total defect density is given by

$$
n=\frac{2}{N} \sum_{k>0} p_{k}(\infty) .
$$

To obtain the standard Kibble-Zurek scaling, in the limit $N \rightarrow$ $\infty$, we can replace the sum in Eq. (31) by an integral and use the asymptotic form of $p_{k}$ given in Eq. (23),

$$
n=\int_{0}^{\pi} \frac{d k}{\pi} p_{k}(\infty)=\int_{0}^{\pi} \frac{d k}{\pi} e^{-\pi J T \sin ^{2}(k)} .
$$

In the adiabatic limit $J T \rightarrow \infty$, only the regions near $k=$ $0, \pi$ contribute to the integral, and we get the Kibble-Zurek scaling law $n \sim T^{-1 / 2}$. This scaling is exactly mirrored by the behavior of the logarithm of the overlap $\mathcal{O}$ in Eq. (27).

As with the overlap, in distinguishing the even and odd sectors, the summation on $k$ in Eq. (31) is restricted to the allowed momenta. The defect density is less sensitive than the overlap in distinguishing between the different topological sectors for the following reason. If the excitation probability $p_{k}(T)$ is close to 1 for any particular value of $k$; this affects the overlap in Eq. (24) strongly since it is given by a product over all $k$ and therefore approaches zero if $1-p_{k}(T)$ is close to zero for any $k$. On the other hand, the defect density in Eq. (31) is given by a sum over all $k$ and is not dominated by any one value of $k$; in addition, the sum is divided by $N$, which further reduces the contribution from any single value of $k$.

For a system of finite-size $N$, in the topologically blocked odd-fermion sector, the special $k=0$ mode has a level crossing and, across the phase transition, completely evolves into the excited state. Compared to the even sector, this mode thus contributes a term of order $1 / N$ independent of the quench rate. In the thermodynamic limit, this contribution obviously vanishes, while away from this limit, the degeneracy in the ferromagnet/topological phase is split due to finite-size effects. However, in this degenerate phase, the splitting is exponentially small as a function of $N$ [44] and is always present in numerical simulations and physical systems due to their finite size. Thus, observation of the quench-independent $1 / N$ jump and its scaling behavior of systems size would provide some indication of the difference between topological sectors.

Residual energy. Another characteristic quantity discussed in quench dynamics is the residual energy; this measures the excess energy contained in a postquench state compared to the ground state of the final Hamiltonian. In the transverse Ising system, the net residual energy at the end of the quench at time $t=T$ is given by the sum of the contributions of each momentum mode,

$$
\mathcal{E}_{\text {res }, k}=\left\langle H_{k}(T)\right\rangle-\mathcal{E}_{k G}(T),
$$

where the expectation value of $H_{k}(T)$ defined in Eq. (15) is with respect to the time-evolved quench state and $\mathcal{E}_{k G}(T)$ is the energy of the ground state of $H_{k}(T)$.

The arguments made above for the defect density also hold for the residual energy. It respects the same $T^{-1 / 2}$ scaling behavior and, in considering the odd- and even-fermion sectors, involves restricted momentum summations. As with the defect density, in the odd-fermion sector the $k=0$ state makes a special contribution, taking the time-evolved state completely into the excited branch. Thus, in this sector, the residual energy shows a jump of order $J$. This too is an effect of order $1 / N$ in that there are contributions from a total of $N$ momentum sets to the entire residual energy. Nevertheless, the jump reflects topological blocking and the difference in behavior of sectors illustrated in Fig. 3.

Entropies. Various forms of entropy, such as the entanglement entropy, have been actively studied in the context of quenches. These measures provide an alternative picture for the manner in which the wave function evolves. In the context of topological sectors, based on the special behavior of the $k=0$ mode, i.e., $p_{k=0}(t>0)=1$, we find that a variant of the Renyi entropy [51], $S_{\alpha}$, would provide an effective way of distinguishing odd and even sectors:

$$
S_{\alpha}=\frac{1}{1-\alpha} \ln \left\{\sum_{k>0}\left[p_{k}(\infty)\right]^{\alpha}\right\} .
$$

Given the Kibble-Zurek scaling form discussed above, $S_{\alpha}$ would behave as $\ln \left[\beta_{o / e}+C(\alpha T)^{-1 / 2}\right]$, where $C$ is a constant and $\beta=0$ for the even-fermion sector, while, in the oddfermion sector, $\beta=1$ is derived from the special $k=0$ mode. By picking $\alpha$ to be large enough, we could force $C(\alpha T)^{-1 / 2} \ll 1$, resulting in $S_{\alpha}$ being close to zero for the odd-fermion sector and large and negative for the even-fermion sector.

An obviously modified version of this discussion of other quench and sector-dependent quantities also holds for the Kitaev model in the next section.

\section{KITAEV'S HONEYCOMB MODEL}

We now explore a model that is truly topological in that while it possesses global topological order and associated degeneracies, it has no local order: the Kitaev honeycomb model [32], shown in Fig. 6 (see also Sec. III B for the full Hamiltonian). The model is very rich in and of itself and has the elegant analytic solution pioneered by Kitaev as well as various alternate analytic approaches.

Before embarking on the relevant details necessary to analyze the Kitaev model in the context of our present work, we first outline how our analysis of the Kitaev model can be understood as a direct two-dimensional extension of the analysis of the previous section. Regardless of whether the reader is familiar with the Kitaev honeycomb, this discussion should make our main results for it clear.

\section{A. A two-dimensional extension of the transverse Ising chain}

In the previous section, we studied the topological description of the Ising chain in terms of a BdG description of a one-dimensional fermionic spinless $p$-wave superconductor in a ring geometry. The Hilbert space was divided into two sectors consisting of momenta that were quantized either according to periodic or antiperiodic boundary conditions and were associated with odd- and even-fermion parities, respectively. Depending on the parameters in the Hamiltonian, the energetics either allowed the two sectors to be degenerate 

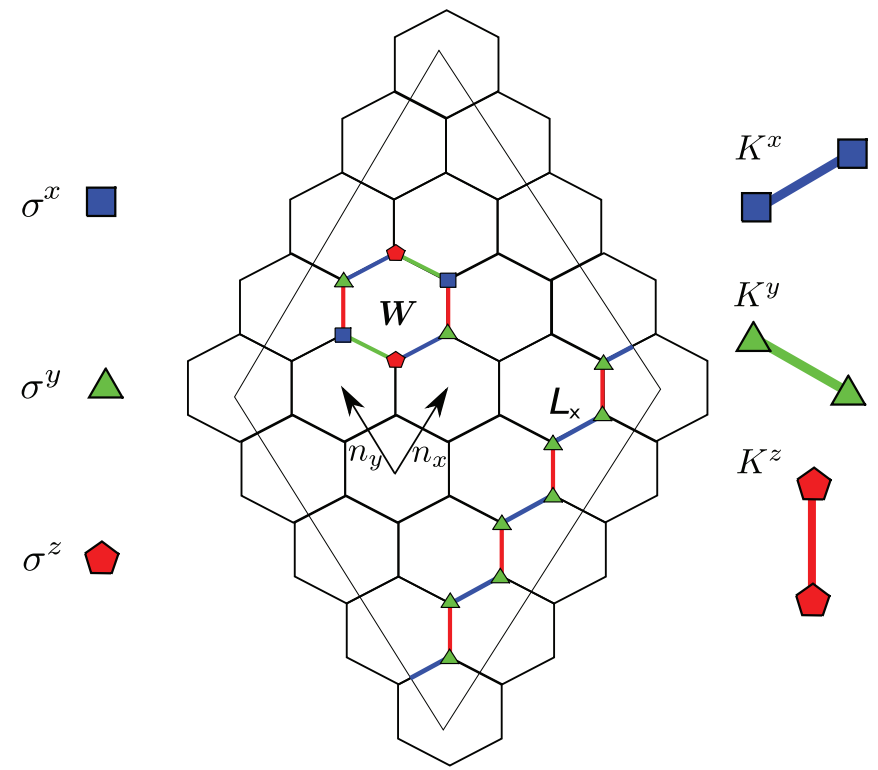

FIG. 6. (Color online) The honeycomb spin model. The $K^{\alpha}$ are directional spin exchange terms which appear in the Hamiltonian (see Section III B). On a torus, we identify opposite sides of the diamond shape. Symmetries in the model are made by making closed product loops of overlapping $K$-terms. The plaquette operators $W$ are the simplest symmetries and exist on the surface of the torus. The homologically non-trivial symmetries (of which we only indicate $L_{x}$ ) are made with overlapping products of $K^{\alpha}$ that loop around the torus.

in ground-state energy or, for the odd sector, to have a higher sectoral ground-state energy than that of the even sector.

With regards to quench dynamics, this mismatch in energy resulted in topological blocking in that if one started in the odd sector in the degenerate phase and quenched into the nondegenerate phase, the overlap with the final absolute ground state would be zero. As for evaluating overlaps between time-evolved quenched states and the final sectoral ground state, this was done by studying the simple dynamics of decoupled pairs of momentum states $\pm \boldsymbol{k}$. The momenta $\boldsymbol{k}=0, \pi$ were special since they respect $\boldsymbol{k}=-\boldsymbol{k}$, and they dictated the fermion parity. The overlaps clearly showed different behavior that depended on the topological (odd/even) sector.

While the Kitaev honeycomb model has several complex, rich aspects, much can be understood by simply generalizing the above to two dimensions. We will see that the Kitaev model can be mapped to a spinless two-dimensional $p$-wave superconductor and the analog of a ring becomes a torus. Topological requirements now dictate periodic or antiperiodic boundary conditions along the two independent ( $x$ and $y$ ) directions, yielding a total of four topological sectors. Unlike in the transverse Ising case, the boundary conditions and fermion parity are not simply related. But in the commonly studied situation where the honeycomb system has no vortices, one which we confine ourselves to, the fermion parity is constrained to be even. As a result, we find that as a function of parameter space, there exist three different phases in which all four sectors have degenerate ground states (Abelian $A$ phases).

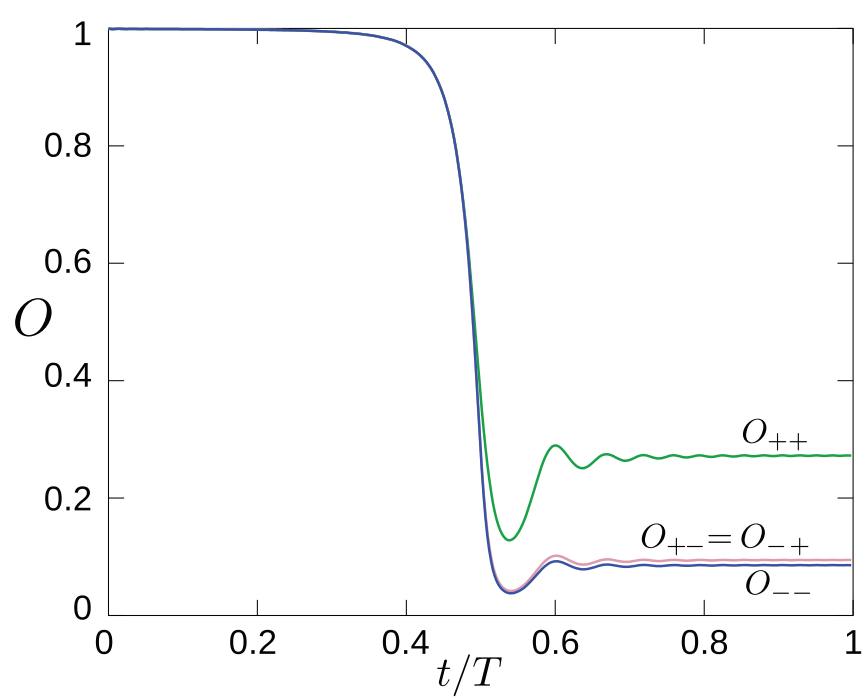

FIG. 7. (Color online) Typical overlap quench data for the Kitaev spin model. In this example we examined a torus of $N_{x}=N_{y}=100$ with a fixed $J_{x}=J_{y}=1, \kappa=0.25$ and time-dependent $J_{z}=3-$ (2t/T) with $T=80$. The blocked sector $(\{++\})$ has a generically higher end overlap. Small differences between the other sectors are due to finite system size.

On the other hand, a fourth phase (non-Abelian $B$ phase) has its absolute ground state in three of the sectors while the fourth sector has higher sectoral ground-state energy.

Thus, similar to the transverse Ising case, topological blocking occurs in one out of the four topological sectors. When evaluating overlaps between time-evolved quenched states and final sectoral ground states, the special momenta are $\left(k_{x}, k_{y}\right)=(0 / \pi, 0 / \pi)$. In Fig. 7 we show the typical overlap data for all four sectors over the course of a quench. By symmetry, two of the time-evolved overlaps $O_{+-}$and $O_{-+}$show identical behaviors. The $O_{--}$overlap is generally different from these other two sectors, but this is a finite-size effect and quickly vanishes for large system sizes. The last overlap $\mathrm{O}_{++}$from the fully periodic sector is distinctly higher than the other three. This is a consequence of topological blocking. In what follows we will explain in more detail the mechanism behind this.

\section{B. Kitaev honeycomb Hamiltonian}

The Kitaev honeycomb system consists of spins on the sites of a hexagonal lattice. The Hamiltonian can be written as

$$
H_{0}=-\sum_{\alpha \in\{x, y, z\}} \sum_{i, j} J_{\alpha} K_{i j}^{\alpha},
$$

where $K_{i j}^{\alpha}=\sigma_{i}^{\alpha} \sigma_{j}^{\alpha}$ denotes a directional spin exchange interaction occurring between sites $i$ and $j$ connected by an $\alpha$ link (see Fig. 6).

Consider now products of $K$ operators along loops on the lattice $K_{i j}^{\alpha^{(1)}} K_{j k}^{\alpha^{(2)}} \cdots K_{l i}^{\alpha^{(n)}}$, where $\alpha^{(m)} \in x, y, z$. Any loop constructed in this way commutes with the Hamiltonian and with all other loops. The shortest such loop symmetries are the plaquette operators

$$
\boldsymbol{W}_{\boldsymbol{q}}=\sigma_{1}^{z} \sigma_{2}^{x} \sigma_{3}^{y} \sigma_{4}^{z} \sigma_{5}^{x} \sigma_{6}^{y},
$$


where the numbers 1 through 6 label lattice sites on a single hexagonal plaquette. We will use the convention that $\boldsymbol{q}$ denotes the $z$ dimer directly below the plaquette. The fact that the Hamiltonian commutes with all plaquette operators implies that we may choose energy eigenvectors $|n\rangle$ such that $w_{q}=$ $\left\langle n\left|\boldsymbol{W}_{\boldsymbol{q}}\right| n\right\rangle= \pm 1$. If $w_{q}=-1$, then we say that the state $|n\rangle$ carries a vortex at $\boldsymbol{q}$. When we refer to a particular vortex sector we mean the subspace of the system with a particular configuration of vortices. The vortex-free sector, for example, is the subspace spanned by all eigenvectors such that $w_{q}=1$ for all $\boldsymbol{q}$.

On a torus of $N$ spins, there are $N / 2$ plaquette $\left(W_{q}\right)$ operators. In general one has the relationship $\prod_{q} W_{q}=I$, so there are $N / 2-1$ independent plaquette operators. We can find two more independent loop operators, which we define as overlapping products of $K^{z}$ and $K^{x}$, or $K^{z}$ and $K^{y}$ operators, which go around homologically nontrivial paths on the torus. We call two such operators, which go through the origin, $L_{x}$ and $L_{y}$, respectively (see Fig. 6). We will see that operators $L_{x}$ and $L_{y}$ play a role similar to the $T_{z}$ operator of the one-dimensional transverse Ising model.

Counting these two operators $L_{x}$ and $L_{y}$ together with the plaquettes $W_{q}$ gives a total of $N / 2+1$ independent symmetries. The different sectors are selected by choosing the respective eigenvalues $l_{x}, l_{y}$, and $w_{q}$. The remaining $N / 2-1$ degrees of freedom are taken up by $N / 2$ fermions (for example, one for each $K^{z}$ link) with the constraint on fermionic parity taken into account.

The breaking of $T$ symmetry is essential for relating the model to a chiral $p$-wave superconductor. Following the work of Ref. [32], we use the three-body term

$$
H_{1}=-\kappa \sum_{q} \sum_{l=1}^{6} P_{q}^{(l)},
$$

with the second summation running over the six terms

$$
\begin{aligned}
\sum_{l=1}^{6} P_{q}^{(l)}= & \sigma_{1}^{x} \sigma_{6}^{y} \sigma_{5}^{z}+\sigma_{2}^{z} \sigma_{3}^{y} \sigma_{4}^{x}+\sigma_{1}^{y} \sigma_{2}^{x} \sigma_{3}^{z} \\
& +\sigma_{4}^{y} \sigma_{5}^{x} \sigma_{6}^{z}+\sigma_{3}^{x} \sigma_{4}^{z} \sigma_{5}^{y}+\sigma_{2}^{y} \sigma_{1}^{z} \sigma_{6}^{x} .
\end{aligned}
$$

For simplicity, in this work we will retain only the terms $P^{(1)}, P^{(2)}, P^{(3)}$, and $P^{(4)}$.

\section{Fermionized solution and phase diagram}

The Kitaev honeycomb Hamiltonian can be solved in several different ways. The method implicitly adopted here is the fermionization procedure used in Refs. $[35,36]$. The procedure involves expressing the $z$ dimers in terms of hardcore bosons and effective spins and then employing string operators to convert bosonic operators to fermionic ones. Importantly, we can associate the presence of a fermion with an antiferromagnetic configuration of the $z$ dimer.

In the $J_{z} \gg J_{x}, J_{y}$ limit, the ground-state manifold contains no fermions (spins connected by a $z$ link point in the same direction). The remaining degrees of freedom are specified through the eigenvalues of the plaquette operators $W$ and the loop operators $L_{x}$ and $L_{y}$. It was shown by Kitaev (see Ref. [32]) that this manifold can be perturbatively mapped on the fourth order to a toric code Hamiltonian

$$
H_{T C}=E_{0}-\frac{J_{x}^{2} J_{y}^{2}}{16\left|J_{z}\right|^{3}} \sum Q_{q},
$$

with $Q_{q}=P\left[W_{q}\right]$, where $P$ is the projector to the ferromagnetic subspace. In this limit, because the projector preserves the eigenvalues of $W$ and $Q$ and because the operators $L_{x}$ and $L_{y}$ do not appear, there are four ground states (labeled by the eigenvalues $l_{x}$ and $l_{y}$ ) with no vortices. As the relative values of $J_{x}$ and $J_{y}$ become larger, the ground states acquire nonzero fermionic components. However, the overall parity of these states cannot change, and it can be proved that the ground states are always vortex free [52]. Hence, given that the zero-vortex sector in the toric code limit has no fermions, in the full Kitaev model, this sector, which contains the ground state, has even parity.

In the vortex-free sector of the Kitaev model, $w_{q}=1 \forall \boldsymbol{q}$, and the associated translationally invariant Hamiltonian can be expressed in momentum space. In terms of fermionic momentum-space operators $c_{k}$, the Hamiltonian takes the BdG form [36]

$$
H=\frac{1}{2} \sum_{k}\left[\begin{array}{ll}
c_{k}^{\dagger} & c_{-k}
\end{array}\right] H_{k}\left[\begin{array}{c}
c_{k} \\
c_{-k}^{\dagger}
\end{array}\right],
$$

with

$$
H_{k}=\left[\begin{array}{cc}
\xi_{k} & \Delta_{k} \\
\Delta_{k}^{*} & -\xi_{k}
\end{array}\right]
$$

where

$$
\begin{aligned}
\xi_{k} & =\varepsilon_{k}-\mu, \\
\Delta_{k} & =\alpha_{k}+i \beta_{k},
\end{aligned}
$$

and

$$
\begin{aligned}
\mu & =-2 J_{z}, \\
\varepsilon_{\boldsymbol{k}} & =2 J_{x} \cos \left(k_{x}\right)+2 J_{y} \cos \left(k_{y}\right), \\
\alpha_{\boldsymbol{k}} & =4 \kappa\left[\sin \left(k_{x}\right)-\sin \left(k_{y}\right)\right], \\
\beta_{\boldsymbol{k}} & =2 J_{x} \sin \left(k_{x}\right)+2 J_{y} \sin \left(k_{y}\right) .
\end{aligned}
$$

Here, $\boldsymbol{k}$ denotes the two-dimensional vector given by momentum components $\left(k_{x}, k_{y}\right)$. Thus, the Kitaev honeycomb system maps to a spinless fermionic BdG Hamiltonian, which, when compared to that associated with the transverse Ising chain in the previous system, can be regarded as a two-dimensional extension. All terms in $\Delta_{k}$ carry net angular momentum $l=1$, and thus the superconducting gap is of $p$-wave nature. The three-body terms in Eqs. (37) and (38) can be seen to open the gap in the $B$ phase of the model and provide a $T$-symmetry-breaking component that makes the system chiral.

As in Sec. II A for the one-dimensional case, we diagonalize the BdG Hamiltonians $H_{k}$ by defining the Bogoliubov-Valatin operators

$$
\begin{aligned}
& \gamma_{k}=u_{k} c_{k}-v_{k} c_{-k}^{\dagger}, \\
& \gamma_{k}^{\dagger}=u_{k}^{*} c_{k}^{\dagger}-v_{k}^{*} c_{-k},
\end{aligned}
$$


with

$$
\begin{aligned}
& u_{k}=\sqrt{\left(1+\xi_{k} / \epsilon_{k}\right) / 2}, \\
& v_{k}=-\sqrt{\left(1-\xi_{k} / \epsilon_{k}\right) / 2} e^{i \arg \left(\Delta_{k}\right)} .
\end{aligned}
$$

As with the one-dimensional case, the modes with $k_{x}, k_{y}=0$ and $\pi$ require a special analysis since they satisfy $k=-k$. Further, $\Delta_{k}=0$ for these modes; hence, $\varepsilon_{k}=\left|\xi_{k}\right|$. The diagonalized Hamiltonian once more takes the form

$$
\begin{aligned}
H & =\sum_{k} \epsilon_{k}\left(\gamma_{k}^{\dagger} \gamma_{k}-1 / 2\right), \\
\epsilon_{k} & =\sqrt{\xi_{k}^{2}+\left|\Delta_{k}\right|^{2}} .
\end{aligned}
$$

The ground state of this has the BCS form,

$$
|\mathrm{gs}\rangle=\prod_{k}\left(u_{k}+v_{k} c_{k}^{\dagger} c_{-k}^{\dagger}\right)|\mathrm{vac}\rangle
$$

which is annihilated by all the $\gamma_{k}$, and has the energy $E_{\mathrm{gs}}=$ $-\frac{1}{2} \int \epsilon_{\boldsymbol{k}} d \boldsymbol{k}$.

The form of the dispersion in Eq. (46) enables us to derive the phase boundaries and gapped/gapless nature of the phases in the honeycomb system. We assume that $J_{x}, J_{y}, J_{z}>0$. As we mentioned above, with this convention the $c$ fermions are associated with antiferromagnetic configurations of the $z$ dimers, and our vacua are toric code states on an effective square lattice [36].

We first consider the case $\kappa=0$; then Eqs. (43) are the same as those used in previous work involving quenches in the Kitaev honeycomb model, namely, Ref. [9] with $\vec{M}_{1}=k_{x}$ and $\vec{M}_{2}=-k_{y}$. From the dispersion, it can be seen that the system is gapless in the range $\left|J_{x}-J_{y}\right|<J_{z}<J_{x}+J_{y}$ and, by symmetry, within similar constraints on $J_{x}$ and $J_{y}$. Thus, as was originally discussed by Kitaev, the system has four phases [32]. The system is gapped in three of the phases, $A_{x}, A_{y}$, and $A_{z}$, with $J_{x}>J_{y}+J_{z}, J_{y}>J_{z}+J_{x}$, and $J_{z}>J_{x}+J_{y}$, respectively. These are called Abelian phases because the low-energy excitations satisfy Abelian statistics under exchanges. In the fourth phase, called $B$, each $J_{i}$ is less than the sum of the other two couplings. The spectrum is gapless in this phase. (This makes it difficult to compute the statistics of the low-energy excitations since even a slow exchange of two of them inevitably produces other low-energy excitations). For instance, if $J_{x}=J_{y}=J>J_{z} / 2$ and $\kappa=0$, we find that the spectrum is gapless at two points given by $k_{x}=-k_{y}= \pm \cos ^{-1}\left(-J_{z} / 2 J\right)$. The spectrum close to these points has the gapless Dirac form with the Dirac cones touching at those points.

If we now make $\kappa \neq 0$, phase $B$ also becomes gapped, with the minimum gap occurring at the two points mentioned above if $\kappa$ is small. The low-energy vortex excitations in this phase are then found to satisfy non-Abelian statistics.

The four phases are separated by quantum phase transition lines on which one of the $J_{i}$ is equal to the sum of the other two couplings. As is standard, the four phases can be depicted in the triangular phase diagram shown in Fig. 8.

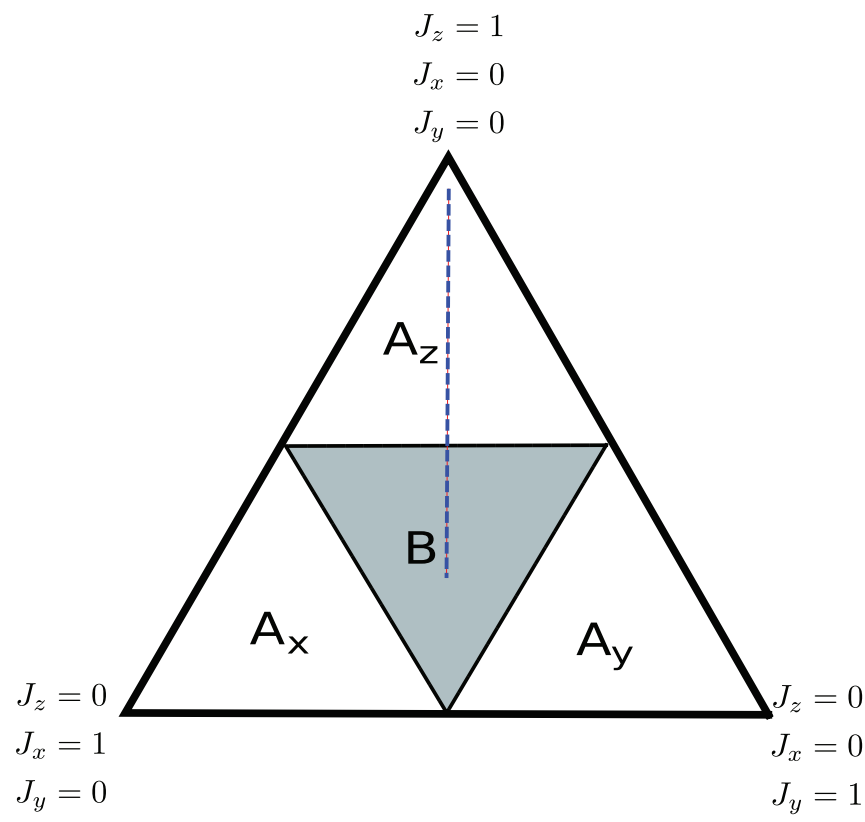

FIG. 8. (Color online) Schematic of the Kitaev model phase diagram. The $A$ regions correspond to toric code phases, while the $B$ region corresponds to the non-Abelian Ising phase. The dashed line indicates the quench parametrization. Note that this diagram uses Kitaev's normalization $J_{x}+J_{y}+J_{z}=1$. In our quench protocol, we use $J_{x}=J_{y}=1$, with $J_{z}$ ranging between 1 and 3 , but on renormalizing, this gives a path similar to the dashed line shown in the diagram.

\section{Topological degeneracy}

The topological nature of the four phases can be directly gleaned by putting the system on a torus. We discussed briefly above how the fourfold degeneracy of the $A$ phases could be understood by mapping perturbatively to the toric code. Let us now see how this looks within the exact fermionic solution of the model where we can also understand the threefold degeneracy of the non-Abelian phase and the gapless nature of the blocked sector.

We remark here that while most of the analysis for the transverse Ising system can be extended into two dimensions for the Kitaev honeycomb system, one crucial difference occurs with regard to fermion parity. In the Ising system, two sectors were allowed based on fermion parity, namely, odd and even sectors, and while these were degenerate in one phase, they were not so in the other. Here, all states in the vortex-free sector have even-fermion-number parity, as argued after Eq. (39). As we shall see below, the degeneracies come about from different combinations of even-fermion occupation.

We assume that the number of sites in the $x$ and $y$ directions are $N_{x}$ and $N_{y}$, with the first site linked to the $N_{i}$ th site along each direction. On the torus, the diagonalized Hamiltonian has a restricted set of momentum modes in its form

$$
H_{=} \sum_{k_{x}, k_{y}} \epsilon_{k}\left(\gamma_{k}^{\dagger} \gamma_{k}-\frac{1}{2}\right),
$$

where the dispersion relation $\epsilon_{\boldsymbol{k}}$ is given in Eq. (46). The allowed values of $k_{\alpha}$ in the various homology sectors on 
the torus are $\theta_{\alpha}+2 \pi \frac{n_{\alpha}}{N_{\alpha}}$ for integer $n_{\alpha}=0,1, \ldots, N_{\alpha}-1$, where the four topological sectors corresponding to $\left(l_{x}, l_{y}\right)=$ $( \pm 1, \pm 1)$ have values of $\theta_{\alpha}$ given by $\theta_{\alpha}=\left(\frac{l_{\alpha}+1}{2}\right) \frac{\pi}{N_{\alpha}}$. The topological sectors dictate whether the wave functions are periodic or antiperiodic. The relationship between the topological sectors and the periodicity/antiperiodicity of the wave functions is simple if a little counterintuitive. For example, the fully periodic sector $(+,+)$ has the quantum numbers $\left(l_{x}, l_{y}\right)=(-1,-1)$, while the fully antiperiodic sector $(-,-)$ has quantum numbers $\left(l_{x}, l_{y}\right)=(1,1)$.

We know that the ground state in the vortex-free sector has even-fermion parity. It can then be shown that in the three topological sectors corresponding to $\left(l_{x}, l_{y}\right)=(1,1),(-1,1)$, and $(1,-1)$, the momenta $\boldsymbol{k}=(0,0),(0, \pi),(\pi, 0)$, and $(\pi, \pi)$ are missing, and the ground state is always of the form given in Eq. (47) with the momenta discretized appropriately as described above. As parity is conserved in each vortex sector, the eigenstates above these ground states are reached by creating fermions in pairs.

In the fully periodic sector $(+,+)$ things are not as straightforward because the four momenta $k=(0,0),(0, \pi),(\pi, 0)$, and $(\pi, \pi)$ are present. Since $\boldsymbol{k}=-\boldsymbol{k}$ and $\Delta_{\boldsymbol{k}}=0$ for all these modes, we have the energetics for these states being purely determined by $\varepsilon_{\boldsymbol{k}}-\mu=2\left[J_{x} \cos \left(k_{x}\right)+J_{y} \cos \left(k_{y}\right)+J_{z}\right]$. In particular, we have $u_{k}=1, v_{k}=0$, and $\gamma_{k}=c_{k}$ if $\varepsilon_{k}-\mu>0$, while $u_{k}=0,\left|v_{k}\right|=1$, and $\gamma_{k}=c_{k}^{\dagger}$ if $\varepsilon_{k}-\mu<0$; in either case $\epsilon_{k}=\left|\varepsilon_{k}-\mu\right|>0$.

The situation in the four different phases and for the four different momenta is summarized in Table I. In the Abelian phase $A_{z}$, where $J_{z}>J_{x}+J_{y}$, we have $\gamma_{k}=c_{k}$ for all four momenta, and we can use the BCS ground state in Eq. (47) where all these momenta are excluded. In the phase $A_{x}$, where $J_{x}>J_{y}+J_{z}$, we have $\gamma_{\boldsymbol{k}} \sim c_{k}^{\dagger}$ for $\boldsymbol{k}=(\pi, 0)$ and $(\pi, \pi)$, so these two momenta must be included as a factor $c_{\pi, 0}^{\dagger} c_{\pi, \pi}^{\dagger}$ in Eq. (47). Similarly, in $A_{y}$, where $J_{y}>J_{z}+J_{x}$, we have $\gamma_{\boldsymbol{k}} \sim c_{\boldsymbol{k}}^{\dagger}$ for $\boldsymbol{k}=(0, \pi)$ and $(\pi, \pi)$, so these momenta must be included as a factor $c_{0, \pi}^{\dagger} c_{\pi, \pi}^{\dagger}$ in Eq. (47). We see that in the three Abelian phases, an even number of momenta are included so that the fermion parity is even as required. We remark here that the asymmetry in the momentum occupation structure between $A_{z}$ and $A_{x}$ and $A_{y}$ can be traced back to the original transformations of the honeycomb Hamiltonian involving dimerization in the $z$ bonds. While this structure is basis dependent, topological aspects, such as degeneracies, are not.

TABLE I. The special momenta $\boldsymbol{k}$ for which $\Delta_{\boldsymbol{k}}=0$ and either $\varepsilon_{k}-\mu>0, \gamma_{k}=c_{k}$ or $\varepsilon_{k}-\mu<0, \gamma_{k}=c_{k}^{\dagger}$ in the four phases $A_{x}, A_{y}, A_{z}$, and $B$. This structure belonging to the fully periodic topological sector determines the ground-state degeneracies of each of the phases.

\begin{tabular}{lcc}
\hline \hline Phase & $\varepsilon_{\boldsymbol{k}}-\mu>0$ & $\varepsilon_{\boldsymbol{k}}-\mu<0$ \\
\hline$A_{x}$ & $(0,0),(0, \pi)$ & $(\pi, 0),(\pi, \pi)$ \\
$A_{y}$ & $(0,0),(\pi, 0)$ & $(0, \pi),(\pi, \pi)$ \\
$A_{z}$ & $(0,0),(\pi, 0),(0, \pi),(\pi, \pi)$ & \\
$B$ & $(0,0),(\pi, 0),(0, \pi)$ & $(\pi, \pi)$ \\
\hline \hline
\end{tabular}

In the $B$ phase, we have $\gamma_{\pi, \pi}=c_{\pi, \pi}^{\dagger}$. However, we cannot include a factor of $c_{\pi, \pi}^{\dagger}$ in Eq. (47) by itself since this would make the fermion parity odd. In Ref. [36] it was demonstrated that the states

$$
|\psi\rangle_{0}=\prod_{k \neq(\pi, \pi)}\left(u_{k}+v_{k} c_{k}^{\dagger} c_{-k}^{\dagger}\right)|\mathrm{vac}\rangle
$$

and

$$
|\psi\rangle_{\boldsymbol{k}^{\prime}}=c_{\pi, \pi}^{\dagger} \gamma_{\boldsymbol{k}^{\prime}}^{\dagger} \prod_{\boldsymbol{k} \neq(\pi, \pi)}\left(u_{\boldsymbol{k}}+v_{\boldsymbol{k}} c_{\boldsymbol{k}}^{\dagger} c_{-\boldsymbol{k}}^{\dagger}\right)|\mathrm{vac}\rangle
$$

can be used as replacements in this scenario, where $\boldsymbol{k}^{\prime}$ can be any momentum apart from $(\pi, \pi)$, thus forming a band. Since the dispersion relation is gapped, all of these states have a higher energy than the ground states of the other three topological sectors.

We can summarize the situation in the thermodynamic limit $N_{x}, N_{y} \gg 1$ as follows. In the three Abelian phases, the ground states in all four topological sectors are degenerate with each other; hence the ground state of the system has a fourfold degeneracy. However, in the $B$ phase, the ground states in the three topological sectors with $\left(l_{x}, l_{y}\right)=(1,1),(-1,1)$, and $(1,-1)$ are degenerate with each other, while the ground state in the sector $\left(l_{x}, l_{y}\right)=(-1,-1)$ has a higher energy; hence the ground state of the system has a threefold degeneracy. The situation is similar to the transverse Ising model where the ground state has a twofold degeneracy in the ferromagnetic phase and has no degeneracy in the paramagnetic phase. We therefore expect that the Kitaev model on a torus will also exhibit topological blocking when the parameters in the Hamiltonian are quenched so as to take it from any one of the Abelian phases to the $B$ phase.

\section{E. Quenching dynamics}

We will now study the quenching dynamics on a torus and discuss the ground-state overlap $O_{ \pm, \pm}(t)$ for each of the four topological sectors as we quench from one of the Abelian phases through a phase transition line into the $B$ phase. As in the previous section, we first discuss topological blocking, then the qualitative features for the time evolution of momentum modes, and then detailed numerical results.

Topological blocking in this case is, in principle, also straightforward (see Fig. 9). The ground state with $\left(l_{x}, l_{y}\right)=$ $(-1,-1)$, which exists in the $A$ phases, does not have a counterpart ground state in the $B$ phase, but the topological quantum numbers $l_{x}$ and $l_{y}$ are conserved in a quench. Therefore, when quenching from the $\left(l_{x}, l_{y}\right)=(-1,-1)$ ground state of an $A$ phase into the $B$ phase, the system is blocked from reaching any of the ground states of the $B$ phase. Understanding this blocking in terms of the occupation of momentum modes is more subtle in the Kitaev honeycomb system than in the transverse Ising system, as can be surmised from the discussion of degeneracies in the previous section. In the three sectors having $\left(l_{x}, l_{y}\right)=(1,1),(-1,1)$, and $(1,-1)$, nothing strange occurs since the four special momenta do not exist in those sectors. But in the sector $(+,+)$ where $\left(l_{x}, l_{y}\right)=(-1,-1)$, the four momenta exist, and they do not evolve at all with time as they do not mix with any other momenta (since $\Delta_{k}=0$ for these modes). We can now understand what will happen 


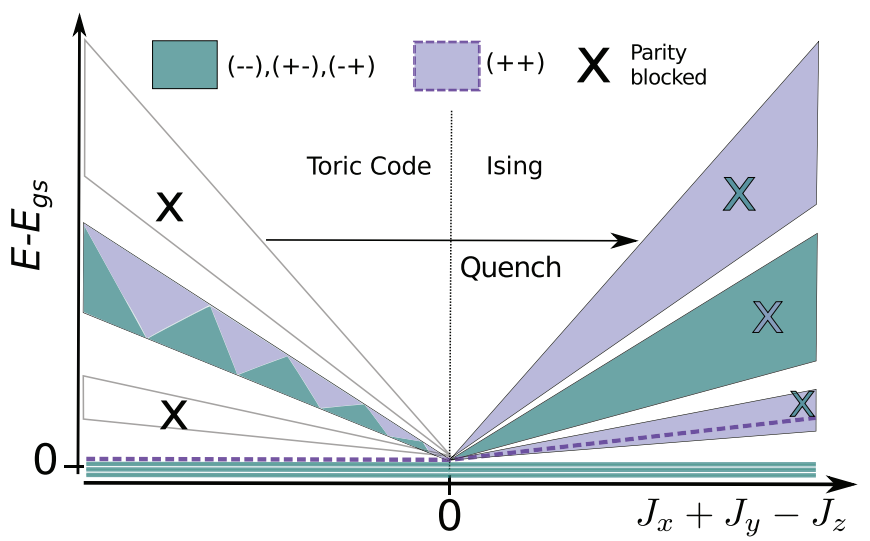

FIG. 9. (Color online) Schematic of the spectrum of the vortexfree sector of the honeycomb model as a function of $J_{x}+J_{y}-J_{z}$. In the toric code phase $\left(J_{z}>J_{x}+J_{y}\right)$, the ground state is fourfold degenerate in the thermodynamic limit with all states being constructed from an even number of fermion excitations. The excitation spectrum consists of bands of states created from the four ground states using pairs of $\gamma^{\dagger}$ operators. As in the transverse Ising case, there are no energy levels with an odd number of $\gamma^{\dagger}$ excitations over one of the ground states. In the non-Abelian Ising phase $\left(J_{z}<J_{x}+J_{y}\right)$, there is a threefold degenerate ground state. The parity-blocking mechanism means that the fully periodic sector is gapped away from the other three states and that the lowest-lying states in this sector form part of a band. Further bands are created from the ground states and this lowest band using pairs of $\gamma^{\dagger}$ operators. The purple dashed line indicates that, in the adiabatic limit, the $(\{++\})$ sectoral ground state in the toric code phase flows into this lowest band but does not necessarily flow to the lowest-energy state in the $(\{++\})$ sector of the Ising phase (the lowest state in the band usually occurs at a different momentum from the initial state, and our quench conserves momentum).

to the ground states of the Abelian phases as we quench into the $B$ phase by looking at Table I. If we start in the ground state of $A_{x}$, which has the modes with momenta $(\pi, 0)$ and $(\pi, \pi)$ occupied, and we quench across the line $J_{x}=J_{y}+J_{z}$, we will reach a low-lying state of the $B$ phase which still has these modes occupied. The lowest-energy state we can reach is the state in Eq. (50) with $\boldsymbol{k}^{\prime}=(\pi, 0)$. If we start in the ground state in $A_{y}$, which has the modes with momenta $(0, \pi)$ and $(\pi, \pi)$ occupied, and we quench across the line $J_{y}=J_{z}+J_{x}$, we will again reach a low-lying state of the $B$ phase with these modes still occupied. The lowest-energy state we can reach is now the state in Eq. (50) with $\boldsymbol{k}^{\prime}=(0, \pi)$. Finally, if we start in the ground state of phase $A_{z}$, the mode with momentum $(\pi, \pi)$ is unoccupied. If we then quench into phase $B$, the lowest state of the $B$ sector that we can reach is the state in Eq. (49). Of course none of the states we reach in this way are ground states of the $B$ phase: the actual ground states are in the other $\left(l_{x}, l_{y}\right)$ sectors. In fact, the lowest states we can reach are not even the lowest-energy states of the low-lying band in the $(+,+)$ sector in the $B$ phase. This is because during the quench, momentum is conserved (the Hamiltonian is always translationally invariant in space), and the lowest states in this band occur at different momenta than the ground states of the $A$ phases in the $(+,+)$ sector. Of course, by changing the details of the quench, breaking translational invariance, we should be able to arrange that the system will flow into the lowest states of the band, but unless we introduce nonlocal perturbations, we will not be able to change the quantum numbers $l_{x}$ and $l_{y}$.

In light of the above discussions, we now study quench dynamics within different topological sectors by initializing the system to a ground state in one of the $A$ phases and quenching through a critical point into the $B$ phase. Our specific quench protocol respects the following evolution:

$$
\begin{aligned}
J_{x} & =J_{y}=J, \quad \kappa=0.1 J, \\
J_{z}(t) & =J(3-2 t / T), \quad 0<t<T .
\end{aligned}
$$

Thus, we start at $t=0$ at $J_{z}=3 J$, which lies in the $A_{z}$ phase, and we end at $t=T$ at $J_{z}=J$, which lies exactly in the middle of the $B$ phase. The phase transition occurs at $t=T / 2$ when $J_{z}=J_{x}+J_{y}$. We note that the since the Hamiltonian conserves the quantum numbers $\left(l_{x}, l_{y}\right)$, the calculations in the different sectors are independent of each other, and there is no mixing between topological sectors.

The analysis of the quenching problem here is very similar in principle to that in the transverse Ising model. Each pair of values $\boldsymbol{k}$ and $\boldsymbol{- k}$ forms a coupled two-level system having Landau-Zener-type dynamics, save for the four special momenta $(0,0),(0, \pi),(\pi, 0)$, and $(\pi, \pi)$, which require a special analysis as we have discussed above. The dynamic Hamiltonian appropriate for each two-level set takes the form

$$
H(t)=J\left(\begin{array}{cc}
-4\left(t-a_{k}\right) / T & b_{k} \\
b_{k}^{*} & 4\left(t-a_{k}\right) / T
\end{array}\right),
$$

where

$$
\begin{aligned}
& a_{\boldsymbol{k}}=\frac{T}{2}\left[3+\cos \left(k_{x}\right)+\cos \left(k_{y}\right)\right], \\
& b_{\boldsymbol{k}}=(2 i+4 \kappa) \sin \left(k_{x}\right)+(2 i-4 \kappa) \sin \left(k_{y}\right) .
\end{aligned}
$$

During the quench, the gap goes through zero only near $\boldsymbol{k}=(\pi, \pi)$. Hence, if $J T \gg 1$, the transition probability $p_{\boldsymbol{k}}$ will differ substantially from zero only in that momentum region. Since the initial and final times $-a_{k}$ and $T-a_{k}$ are approximately given by $-T / 2$ and $T / 2$ and $T$ is large, we can use the expression in Eq. (23) for $p_{\boldsymbol{k}}$. Assuming that $N_{x}=N_{y}=N \gg 1$, we define the variable $\bar{T}=\pi^{2} J T / N^{2}$. Expanding the momentum around $\boldsymbol{k}=(\pi, \pi)$, we have the following expressions in the four topological sectors:

$$
\left(\pi-k_{x}, \pi-k_{y}\right)= \begin{cases}\frac{\pi}{N}\left(2 n_{x}+2,2 n_{y}+2\right) & \text { in }(+,+), \\ \frac{\pi}{N}\left(2 n_{x}+2,2 n_{y}+1\right) & \text { in }(+,-), \\ \frac{\pi}{N}\left(2 n_{x}+1,2 n_{y}+2\right) & \text { in }(-,+), \\ \frac{\pi}{N}\left(2 n_{x}+1,2 n_{y}+1\right) & \text { in }(-,-),\end{cases}
$$

where $n_{x}=0,1, \ldots, N / 2-1$ and $n_{y}=-N / 2,-N / 2+$ $1, \ldots, N / 2-1$. We have chosen these ranges of $n_{x}$ and $n_{y}$ in such a way that for each pair of values $\boldsymbol{k}$ and $-\boldsymbol{k}$, exactly one value of $\boldsymbol{k}$ appears in Eq. (54).

At this point we recall the subtlety of topological blocking in the fully periodic sector $(+,+)$ of the Kitaev model. Namely, if we start in any of the $A$ phases, a quench through the critical point will not take us to the ground state of the $B$ phase because one of the four special momenta $[(0,0),(\pi, 0)$, $(0, \pi)$, and $(\pi, \pi)]$ will fail to change to its appropriate ground or excited state. 
Using Eqs. (54) to write $\boldsymbol{k}$ in terms of $n_{x}, n_{y}$, we find that the overlaps between the ground state and the state reached at the final time $t=T$ in the different sectors are given by

$$
\begin{aligned}
\mathcal{O}_{ \pm, \pm}(T) & =\prod_{n_{x}=0}^{\infty} \prod_{n_{y}=-\infty}^{\infty}\left(1-p_{n_{x}, n_{y}}\right), \\
p_{n_{x}, n_{y}} & =e^{-\pi J T\left|b_{n_{x}, n_{y}}\right|^{2} / 4} \\
& =\left\{\begin{array}{l}
e^{-\pi \bar{T}\left[\left(2 n_{x}+2 n_{y}+4\right)^{2}+4 \kappa^{2}\left(2 n_{x}-2 n_{y}\right)^{2}\right]} \text { in }(+,+), \\
e^{-\pi \bar{T}\left[\left(2 n_{x}+2 n_{y}+3\right)^{2}+4 \kappa^{2}\left(2 n_{x}-2 n_{y}+1\right)^{2}\right]} \text { in }(+,-), \\
e^{-\pi \bar{T}\left[\left(2 n_{x}+2 n_{y}+3\right)^{2}+4 \kappa^{2}\left(2 n_{x}-2 n_{y}-1\right)^{2}\right]} \text { in }(-,+), \\
e^{-\pi \bar{T}\left[\left(2 n_{x}+2 n_{y}+2\right)^{2}+4 \kappa^{2}\left(2 n_{x}-2 n_{y}\right)^{2}\right]} \text { in }(-,-) .
\end{array}\right.
\end{aligned}
$$

Note that we have changed the upper limit for $n_{x}$ from $N / 2-1$ to $\infty$ and the limits for $n_{y}$ from $[-N / 2-1, N / 2]$ to $[-\infty, \infty]$; this is justified for large values of $T$ since the overlap $1-$ $p_{n_{x}, n_{y}}(T)$ rapidly approaches 1 once $n_{x} / N,\left|n_{y}\right| / N$ become numbers of order 1 . For $4 \kappa^{2}<1$, a term-by-term comparison shows that $\mathcal{O}_{+,+}>\mathcal{O}_{+,-}=\mathcal{O}_{-,+}>\mathcal{O}_{-,-}$.

As in the transverse Ising model, the log of the overlaps can be written as sums over $n_{x}, n_{y}$, which can then be written as integrals in the limit $\bar{T} \rightarrow 0$. Ignoring the integers $1,2,3,4$ in Eqs. (55), which amounts to ignoring some subleading terms, we find that

$$
\begin{aligned}
\log & \mathcal{O}(T) \\
= & \int_{0}^{\infty} \int_{-\infty}^{\infty} d n_{x} d n_{y} \log \left(1-e^{-4 \pi \bar{T}\left[\left(n_{x}+n_{y}\right)^{2}+4 \kappa^{2}\left(n_{x}-n_{y}\right)^{2}\right]}\right) \\
= & -\frac{\pi^{2}}{192 \kappa \bar{T}}
\end{aligned}
$$

in all four topological sectors. We observe that this diverges if $\kappa \rightarrow 0$; this is because in this limit, the off-diagonal element in the Hamiltonian in Eq. (52) vanishes at not just the four special momenta but along the entire line in the Brillouin zone given by $k_{y}=-k_{x}$. The space of momenta for which $p_{k}=1$ is therefore no longer zero-dimensional but instead one-dimensional. Looking at the expressions in Eqs. (55) and (56), we see that if $\kappa=0$, the integral over $n_{x}+n_{y}$ gives a factor of order $1 / \sqrt{\bar{T}}$, while the integral over $n_{x}-n_{y}$ gives a factor of order $N$. We therefore expect that $\log \mathcal{O}(T)$ will be of order $N^{2} / \sqrt{J T}$ if $\kappa=0$; this is in contrast to the term of order $1 /(\kappa \bar{T}) \sim N^{2} /(\kappa J T)$ that we get if $\kappa \neq 0$. We note here that the Kibble-Zurek power law for the defect density is known [9] to have a similar dependence on $\kappa$; the power law changes from $T^{-1 / 2}$ for $\kappa=0$ to $T^{-1}$ for $\kappa \neq 0$.

As in the transverse Ising model, the overlaps $\mathcal{O}_{ \pm, \pm}(t)$ show oscillations around $t=T / 2$ for a certain range of values of $\bar{T}$. As before, this can be understood by looking at the overlaps for individual values of $\boldsymbol{k}$ lying in the region close to $(\pi, \pi)$. For $\bar{T} \ll 1$, the overlap changes quickly from 1 to small values for several values of $\boldsymbol{k}$ in that region; hence, the overlap of the system (which is given by the product of the overlaps over all values of $\boldsymbol{k}$ ) changes rapidly from 1 to a very small value. For $\bar{T} \gg 1$, the overlap remains close to 1 for all values of $\boldsymbol{k}$; hence, the overlap of the system remains close to 1 .
Thus, the overlap shows noticeable oscillations near $t=T / 2$ only if $\bar{T}$ has a value of order 1 in such a way that only the value of $\boldsymbol{k}$ lying closest to $(\pi, \pi)$ [this corresponds to $n_{x}=$ $n_{y}=0$ in Eqs. (55)] has $p_{k}(t)$ varying substantially with $t$ and all other values of $\boldsymbol{k}$ have $p_{\boldsymbol{k}}(t) \approx 1$ for all $t$. Using the expressions for $p_{n_{x}, n_{y}}$ in Eqs. (55) and setting $p_{0,0}=0.5$, we find that the values of $\bar{T}$ where $\mathcal{O}_{ \pm, \pm}(T) \approx 0.5$ are given by $0.22 / 16 \approx 0.014$ in the sector $(+,+), 0.22 /\left(9+4 \kappa^{2}\right)(\approx 0.024$ for $\kappa=0.1)$ in sectors $(+,-)$ and $(-,+)$, and $0.22 / 4=0.055$ in the sector $(-,-)$. These numbers also provide estimates of the values of $\bar{T}$ where the oscillations around $t=T / 2$ are most prominent.

In Fig. 7 we show the numerically calculated overlaps for the four sectors of the honeycomb model. The figure was calculated using the two-dimensional equivalent of Eq. (20) and clearly illustrates the predicted sectoral dependence of the overlap in quenching from the $A$ phase into the $B$ phase. We note, however, that $O_{++}$is the overlap between the timeevolved state and the state that would be reached by adiabatic transport of the initial state (that is, the lowest-energy state in this sector with the same momentum). In this case, unlike the blocked sector of the transverse Ising model, the adiabatically transported state is not the lowest-energy state in the band (see Fig. 9).

In Fig. 10, we compare the scaling of the final overlap $\mathcal{O}_{++}(T)$ obtained numerically versus the scaling predicted in Eq. (56). We see that the agreement is good at large values of $N^{2} / T$ (where $N^{2}=N_{x} N_{y}$ ), but there are some deviations at small values of $N^{2} / T$. The reason for the latter is as follows. In going from Eq. (55) to (56) for the log of the overlap, we have replaced the sums over $n_{x}, n_{y}$ by integrals. This is justifiable only if the terms being summed over vary slowly with $n_{x}, n_{y}$. However, if $\bar{T}=\pi^{2} J T / N^{2}$ is large, we can see from Eq. (55) that the terms vary rapidly with $n_{x}, n_{y}$, going to zero quickly as $n_{x}, n_{y}$ increase.

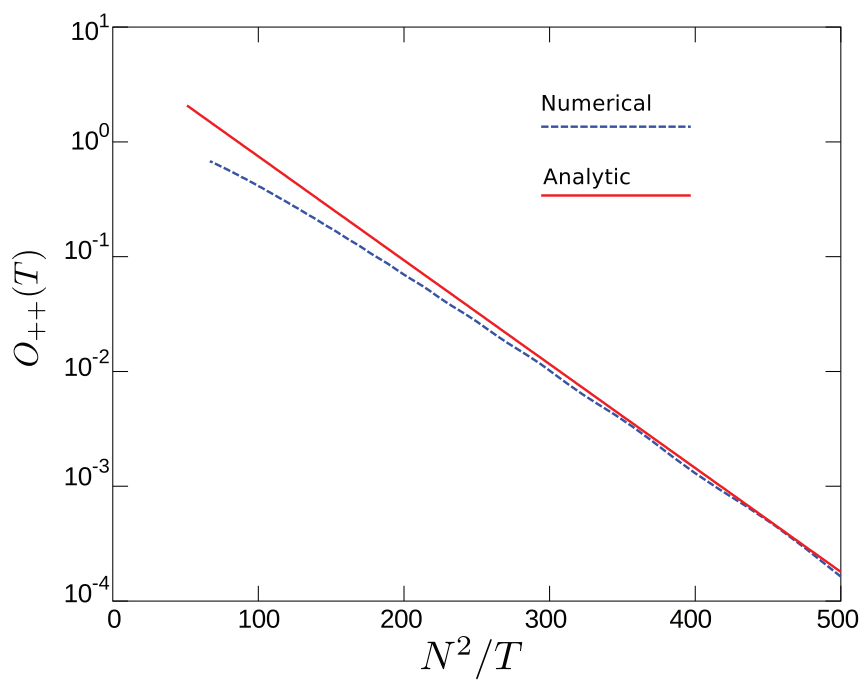

FIG. 10. (Color online) The numerical scaling of the final overlap as a function of $N^{2} / T$ against the predicted behavior in Eq. (56). The system size used here is $N_{x}=N_{y}=100$ with $J_{x}=J_{y}=1, \kappa=0.25$, and $J_{z}=3-(2 t / T)$. 


\section{DISORDER EFFECTS}

So far, we have discussed topological blocking and the dependence of the quench behavior of overlaps on topological sectors in fermion/spin models that preserve translational invariance. However, despite the crucial difference between the unblocked and blocked sectors (the former is gapped, while there exists a gapless spectrum above the sectoral ground state of the blocked sector), one does not observe any real qualitative difference in the postquench overlap behavior. This is because, even in the gapless sector, there is an effective gap to the lowest-energy excited state with the same momentum as the ground state. However, a disordered quench will mix these different momenta, and thus, we can then observe the major characteristic differences between the quenches in the different topological sectors.

Here, we bring out this qualitative difference by analyzing a disordered version of the transverse Ising system. Our disordered quench protocol, which we numerically implement in the one-dimensional case, involves explicitly randomizing in position space the quench term in Eq. (14) as $h_{i}(t)=h(t)+V_{i}$. Here $V_{i}$ are random values from a Gaussian distribution with standard deviation $\sigma$. We keep this additional term fixed for the duration of the quench. It is important to note that in the presence of disorder the eigenvalues of the operator $T_{z}$ are still good quantum numbers even though we cannot directly associate them with discrete momenta.

In Fig. 11 we show numerical results for overlaps for multiple quenches with different static disorder potentials. Comparing this case with the translationally invariant case (dashed gray line), we see that the behavior of the blocked state overlap is characteristically different immediately after

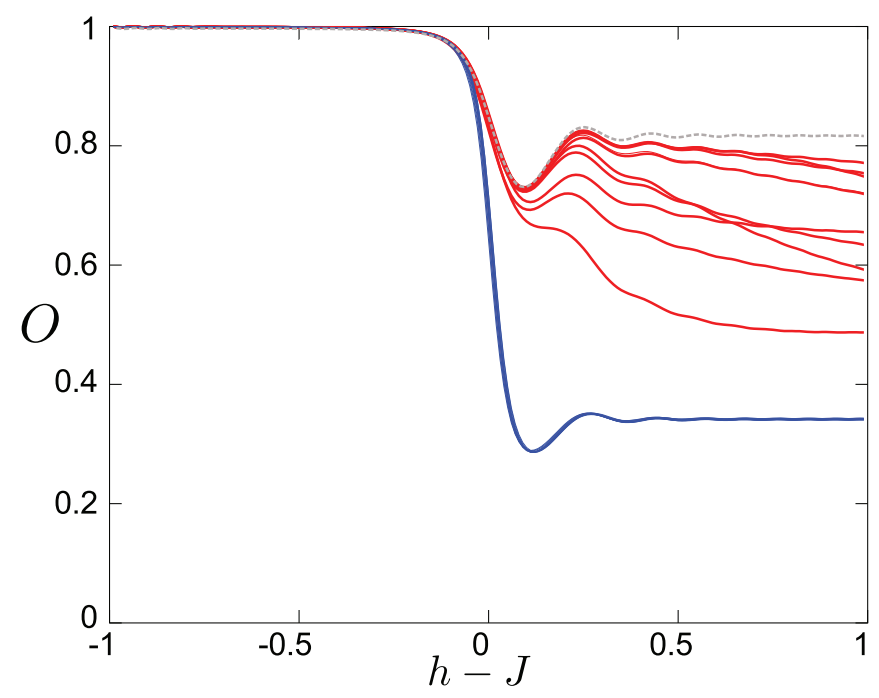

FIG. 11. (Color online) Instantaneous ground-state overlap for a number of disorder configurations which are constant in time. Disorder has a negligible effect on the overlap profile of the unblocked (blue) sector. On the other hand, we see that disorder allows the blocked sector (red) to disperse within the band, inducing a clear instability in the overlap profile. The zero-disorder values are indicated here by the dashed gray line for comparison. Here, we examined a $N=60$ site chain with $J=1$ over a time $T=50$. The disorder configurations used have standard deviations $\sigma=0.1$. the quench. This is a generic phenomenon that we observe for all disorder configurations and indicates clearly the gapless nature of the sector.

In principle, we could have considered disorder in any local quadratic-fermion operator extending over a few lattice sites, and our results would have remained robust. This is because $T_{z}$ commutes with all such operators and thus remains a good quantum number. For example, we could also have randomized the coupling $J$ in position space. The fact that there are still two separate sectors is emphasized in Fig. 11 through the observation that the two overlaps show distinctly different quench behavior. On the other hand, it is important to note that in the spin language, perturbations involving, for example, local $\sigma^{x}$ operators would break the degeneracy of the initial ferromagnetic phase, giving way to a preferred polarization direction. In the fermionic representation we can understand this by noting that these operators break fermion parity and carry with them strings that violate our fermionic notion of local operations.

Finally, it should be noted that in situations like the one described here, where we have some topological sectors with a gap and some sectors which are gapless and have a low-lying band, we can always expect to have considerable differences between the sectors' postquench behaviors at finite temperature. This is obviously relevant to any experimental setting in which such quenches might be performed. Suppose the system is kept in contact with a reservoir at temperature $T$ such that $k T$ is much smaller than the gap in the gapped sectors but considerably larger than the typical energy spacing in the low-lying band in a gapless sector (in the thermodynamic limit, this spacing goes to zero, while the gapped sectors remain gapped). Regardless of the detailed mechanism of energy exchange between the system and the reservoir, one would then expect that in a slow quench starting in one of the blocked sectors, the system will end up in a thermal mixed state involving many of the states in the low-lying band. As a result, it could be observed with a wide range of momenta. On the other hand, in the gapped sectors, contact with a reservoir at some temperature well below the gap should have very little effect. Of course, we may also imagine that the presence of a reservoir will eventually mix states in different topological sectors. But the characteristic time for such equilibration should be much longer than the characteristic time for mixing states within a single sector because the Hamiltonian for the interaction between the system and the reservoir should not depend on the nonlocal quantum numbers characterizing the sectors.

\section{SUMMARY AND OUTLOOK}

In summary, we have explored the notion of topological blocking, which depends on ground-state degeneracies, and quench dependence on topological sectors as concepts that ought to be applicable to most topological systems. We have demonstrated these concepts in the context of topological spinless fermionic $p$-wave systems (analogous to superconductors), derived from the transverse Ising chain in one dimension and the zero-vortex sector of the Kitaev honeycomb model in two dimensions. Confining ourselves to translationally invariant systems and periodic boundary conditions has 
allowed us to study decoupled pairs of momentum modes respecting Bogoliubov-de Gennes Hamiltonians. Topological sectors and degeneracies have been identified in terms of fermion parity, dictated by the occupation numbers of special unpaired momentum modes. In the Ising systems, we have illustrated topological blocking in quenching from the doubledegeneracy topological phase to the nontopological phase with a unique ground state and in the Kitaev honeycomb system from a fourfold degenerate Abelian phase to a threefold degenerate non-Abelian phase. Our analytic treatment of quench within different topological sectors has involved employing Landau-Zener physics within each momentum sector and has been corroborated by numerics. We have found that a sensitive measure of quench dependence on topological sectors is the overlap between the time-evolved initial ground state within a sector and the sectoral ground state of the final Hamiltonian, or, more precisely, the overlap between the time-evolved state and the state that it would evolve to in the adiabatic limit. Finally, by numerically incorporating disorder in our quench protocol, we have shown that quench behavior in different topological sectors can be qualitatively very different, particularly if the blocked sector can access a gapless spectrum.

The mapping in the transverse Ising system between a model having local $\mathbb{Z}_{2}$ symmetry and one with topological order begs for a comment on the relevance of our analyses to systems with spontaneous symmetry breaking and local order. As with topological systems, in quenching through a spontaneous symmetry-breaking transition, the symmetry-broken phase would typically have larger ground-state degeneracy than the unbroken phase, and if the quench dynamics preserves the symmetry, a similar blocking phenomenon can occur; some symmetry-breaking states would be lifted away from the ground-state energy in the unbroken phase. In fact, much of our analysis would apply for these systems, and it would be worth studying sectoral dependences in the context of local order as well. However, an important distinction of topological blocking is the nonlocal nature of topological symmetries. Thus, unlike in spontaneous symmetry-broken systems, the key features of topological blocking discussed in this work should be robust against local perturbations of the Hamiltonian of the system.

This study explicitly addresses degeneracies by way of topological blocking and distinguishing topological sectors via quench dynamics, and there are several avenues for further investigation. Our analyses of the dynamic behavior of overlaps and of other quantities are by no means exhaustive; we hope to develop these further. While our quench protocol has involved a linear quench, several studies have investigated the effect of nonlinear quenches $[10,11]$; it would be worthwhile to ask whether these quenches can highlight topological aspects better than the linear quench. Starting with the original Kibble-Zurek treatments, several works have considered quench physics in terms of defects, vortices, and vortex loops, and it would be interesting to see if these entities have different structures that depend on the topological sectors. As for the treatment of disorder in the last section, our studies are very preliminary. There is scope for an extensive study bringing out qualitative differences between sectors and making connections with other work on disordered quenches (e.g., Refs. [53-55]). In the Kitaev honeycomb system, translational symmetry can also be broken by considering the system away from the zero-vortex sector; the inclusion of vortices amounts to changing signs on the bonds in a lattice model of the $p$-wave superconductor. Such a study could also tie in with predicted vortex-nucleation properties (see, for example, Refs. [56,57]).

Turning to other topological systems of interest, the fractional quantum Hall systems are a paradigmatic example of topological order, extensively studied for their degeneracy properties on the torus. In principle, some of the various techniques used to analyze quantum Hall systems can also be employed to study quenching in the context here. While Abelian states, such as Laughlin states, would perhaps be simpler to analyze, non-Abelian states would be of much interest in the parallels with chiral superconductors $(v=5 / 2)$ [58]. It would also be of great interest to study quenching in systems with symmetry-protected topological order [59,60], such as topological insulators. Experimental settings for studying features discussed here could also include spin chains, the recently realized topological superconducting wires [41], lattice models in cold atomic gases [61-63], and quantum Hall systems.

\section{ACKNOWLEDGMENTS}

We thank A. Chandran, V. Chua, F. Essler, and J. Vala for insightful comments. G.K. acknowledges the financial support of Science Foundation Ireland under the Award No. 10/IN.1/I3013 and the Alexander von Humbolt Foundation, D.S. thanks DST, India, for support under Project No. SR/S2/JCB-44/2010, J.K.S. acknowledges funding from Science Foundation Ireland Grants No. 08/IN.1/I1961 and No. 12/IA/1697, and S.V. thanks the Simons Foundation for support under Grant No. 229047 and the National Science Foundation for support under Grant No. DMR 0644022-CAR. We particularly wish to thank the Aspen Center for Physics, where these studies were initiated.
[1] J. Dziarmaga, Adv. Phys. 59, 1063 (2010); A. Polkovnikov, K. Sengupta, A. Silva, and M. Vengalattore, Rev. Mod. Phys. 83, 863 (2011); A. Dutta, U. Divakaran, D. Sen, B. K. Chakrabarti, T. F. Rosenbaum, and G. Aeppli, arXiv:1012.0653.

[2] T. W. B. Kibble, J. Phys. A 9, 1387 (1976); Phys. Rep. 67, 183 (1980).

[3] W. H. Zurek, Nature (London) 317, 505 (1985); Phys. Rep. 276, 177 (1996).
[4] W. H. Zurek, U. Dorner, and P. Zoller, Phys. Rev. Lett. 95, 105701 (2005); J. Dziarmaga, ibid. 95, 245701 (2005); B. Damski, ibid. 95, 035701 (2005); B. Damski and W. H. Zurek, Phys. Rev. A 73, 063405 (2006).

[5] A. Polkovnikov, Phys. Rev. B 72, 161201(R) (2005); A. Polkovnikov and V. Gritsev, Nat. Phys. 4, 477 (2008).

[6] P. Calabrese and J. Cardy, J. Stat. Mech. (2005) P04010; Phys. Rev. Lett. 96, 136801 (2006). 
[7] R. W. Cherng and L. S. Levitov, Phys. Rev. A 73, 043614 (2006).

[8] V. Mukherjee, U. Divakaran, A. Dutta, and D. Sen, Phys. Rev. B 76, 174303 (2007); U. Divakaran, A. Dutta, and D. Sen, ibid. 78, 144301 (2008); S. Deng, G. Ortiz, and L. Viola, Europhys. Lett. 84, 67008 (2008); U. Divakaran, V. Mukherjee, A. Dutta, and D. Sen, J. Stat. Mech. (2009) P02007; V. Mukherjee and A. Dutta, Europhys. Lett. 92, 37004 (2010).

[9] K. Sengupta, D. Sen, and S. Mondal, Phys. Rev. Lett. 100, 077204 (2008); S. Mondal, D. Sen, and K. Sengupta, Phys. Rev. B 78, 045101 (2008).

[10] D. Sen, K. Sengupta, and S. Mondal, Phys. Rev. Lett. 101, 016806 (2008); S. Mondal, K. Sengupta, and D. Sen, Phys. Rev. B 79, 045128 (2009).

[11] C. De Grandi, R. A. Barankov, and A. Polkovnikov, Phys. Rev. Lett. 101, 230402 (2008).

[12] R. Barankov and A. Polkovnikov, Phys. Rev. Lett. 101, 076801 (2008); C. De Grandi, V. Gritsev, and A. Polkovnikov, Phys. Rev. B 81, 012303 (2010).

[13] D. Patanè, A. Silva, L. Amico, R. Fazio, and G. E. Santoro, Phys. Rev. Lett. 101, 175701 (2008); D. Patanè, L. Amico, A. Silva, R. Fazio, and G. E. Santoro, Phys. Rev. B 80, 024302 (2009).

[14] A. Bermudez, D. Patanè, L. Amico, and M. A. Martin-Delgado, Phys. Rev. Lett. 102, 135702 (2009); A. Bermudez, L. Amico, and M. A. Martin-Delgado, New J. Phys. 12, 055014 (2010).

[15] J. H. H. Perk and H. Au-Yang, J. Stat. Phys. 135, 599 (2009).

[16] D. Sen and S. Vishveshwara, Europhys. Lett. 91, 66009 (2010); W. DeGottardi, D. Sen, and S. Vishveshwara, New J. Phys 13, 065208 (2011).

[17] F. Pollmann, S. Mukerjee, A. G. Green, and J. E. Moore, Phys. Rev. E 81, 020101(R) (2010).

[18] A. Dutta, R. R. P. Singh, and U. Divakaran, Europhys. Lett. 89, 67001 (2010); T. Hikichi, S. Suzuki, and K. Sengupta, Phys. Rev. B 82, 174305 (2010).

[19] A. Chandran, A. Erez, S. S. Gubser, and S. L. Sondhi, Phys. Rev. B 86, 064304 (2012).

[20] A. Chandran, F. J. Burnell, V. Khemani, and S. L. Sondhi, J. Phys. Condens. Matter 25, 404214 (2013).

[21] S. Mostame, C. Castelnovo, R. Moessner, and S. L. Sondhi, Proc. Natl. Acad. Sci. U.S.A. 111, 640 (2014).

[22] M. S. Foster, V. Gurarie, M. Dzero, and E. A. Yuzbashyan, arXiv:1307.2256.

[23] A. A. Patel, S. Sharma, and A. Dutta, Eur. Phys. J. B 86, 367 (2013).

[24] C. Nayak, S. H. Simon, A. Stern, M. Freedman, and S. Das Sarma, Rev. Mod. Phys. 80, 1083 (2008).

[25] J. Alicea, Y. Oreg, G. Refael, F. von Oppen, and M. P. A. Fisher, Nat. Phys. 7, 412 (2011).

[26] S. Vishveshwara, Nat. Phys. 7, 450 (2011).

[27] G. Kells, V. Lahtinen, and J. Vala, Phys. Rev. B 89, 075122 (2014).

[28] E. Barouch, B. M. McCoy, and M. Dresden, Phys. Rev. A 2, 1075 (1970); E. Barouch and B. M. McCoy, ibid. 3, 2137 (1971).

[29] P. Calabrese, F. H. L. Essler, and M. Fagotti, J. Stat. Mech. (2012) P07016.
[30] F. H. L. Essler, S. Evangelisti, and M. Fagotti, Phys. Rev. Lett. 109, 247206 (2012).

[31] M. Fagotti, M. Collura, F. H. L. Essler, and P. Calabrese, Phys. Rev. B 89, 125101 (2014).

[32] A. Kitaev, Ann. Phys. (N.Y.) 321, 2 (2006).

[33] H.-D. Chen and Z. Nussinov, J. Phys. A 41, 075001 (2008).

[34] G. Baskaran, S. Mandal, and R. Shankar, Phys. Rev. Lett. 98, 247201 (2007); D.-H. Lee, G.-M. Zhang, and T. Xiang, ibid. 99, 196805 (2007); Z. Nussinov and G. Ortiz, Phys. Rev. B 77, 064302 (2008).

[35] G. Kells, A. T. Bolukbasi, V. Lahtinen, J. K. Slingerland, J. K. Pachos, and J. Vala, Phys. Rev. Lett. 101, 240404 (2008).

[36] G. Kells, J. K. Slingerland, and J. Vala, Phys. Rev. B 80, 125415 (2009).

[37] K. P. Schmidt, S. Dusuel, and J. Vidal, Phys. Rev. Lett. 100, 057208 (2008); S. Dusuel, K. P. Schmidt, and J. Vidal, ibid. 100, 177204 (2008); J. Vidal, K. P. Schmidt, and S. Dusuel, Phys. Rev. B 78, 245121 (2008).

[38] G. Kells and J. Vala, Phys. Rev. B 82, 125122 (2010).

[39] J. Knolle, D. L. Kovrizhin, J. T. Chalker, and R. Moessner, Phys. Rev. Lett. 112, 207203 (2014).

[40] A. Kitaev, Phys. Usp. 44, 131 (2001).

[41] V. Mourik, K. Zuo, S. M. Frolov, S. R. Plissard, E. P. A. M. Bakkers, and L. P. Kouwenhoven, Science 336, 1003 (2012); M. T. Deng, C. L. Yu, G. Y. Huang, M. Larsson, P. Caroff, and H. Q. Xu, Nano Lett. 12, 6414 (2012); L. P. Rokhinson, X. Liu, and J. K. Furdyna, Nat. Phys. 8, 795 (2012); A. Das, Y. Ronen, Y. Most, Y. Oreg, M. Heiblum, and H. Shtrikman, ibid. 8, 887 (2012); A. D. K. Finck, D. J. Van Harlingen, P. K. Mohseni, K. Jung, and X. Li, Phys. Rev. Lett. 110, 126406 (2013); H. O. H. Churchill, V. Fatemi, K. Grove-Rasmussen, M. T. Deng, P. Caroff, H. Q. Xu, and C. M. Marcus, Phys. Rev. B 87, 241401(R) (2013).

[42] S. Sachdev, Quantum Phase Transitions (Cambridge University Press, Cambridge, 1999).

[43] P. Pfeuty, Ann. Phys. (N.Y.) 57, 79 (1970).

[44] E. Lieb, T. Schultz, and D. Mattis, Ann. Phys. (N.Y.) 16, 407 (1961).

[45] Y. Niu, S. B. Chung, C.-H. Hsu, I. Mandal, S. Raghu, and S. Chakravarty, Phys. Rev. B 85, 035110 (2012).

[46] P. Ring and P. Schuck, The Nuclear Many-Body Problem, 3rd ed. (Springer, Berlin, 2004).

[47] C. Zener, Proc. R. Soc. London, Ser. A 137, 696 (1932); L. D. Landau and E. M. Lifshitz, Quantum Mechanics: Non-relativistic Theory, 2nd ed. (Pergamon, Oxford, 1965).

[48] E. Majorana, Nuovo Cimento 9, 43 (1932); E. C. G. Stueckelberg, Helv. Phys. Acta 5, 369 (1932).

[49] N. V. Vitanov and B. M. Garraway, Phys. Rev. A 53, 4288 (1996).

[50] M. Kolodrubetz, B. K. Clark, and D. A. Huse, Phys. Rev. Lett. 109, 015701 (2012).

[51] P. Calabrese, M. Campostrini, F. Essler, and B. Nienhuis, Phys. Rev. Lett. 104, 095701 (2010).

[52] E. H. Lieb, Phys. Rev. Lett. 73, 2158 (1994).

[53] B. V. Fine, Phys. Rev. E 80, 051130 (2009).

[54] G. P. Brandino, A. De Luca, R. M. Konik, and G. Mussardo, Phys. Rev. B 85, 214435 (2012).

[55] F. Kolley, O. Bohigas, and B. V. Fine, arXiv:1209.2954. 
[56] C. Gils, E. Ardonne, S. Trebst, A. W. W. Ludwig, M. Troyer, and Z. Wang, Phys. Rev. Lett. 103, 070401 (2009).

[57] V. Lahtinen, A. W. W. Ludwig, J. K. Pachos, and S. Trebst, Phys. Rev. B 86, 075115 (2012); V. Lahtinen, A. W. W. Ludwig, and S. Trebst, ibid. 89, 085121 (2014).

[58] N. Read and D. Green, Phys. Rev. B 61, 10267 (2000).

[59] F. Pollmann, E. Berg, A.-M. Turner, and M. Oshikawa, Phys. Rev. B 85, 075125 (2012).
[60] Z.-C. Gu and X.-G. Wen, Phys. Rev. B 80, 155131 (2009).

[61] M. Greiner, O. Mandel, T. W. Hansch, and I. Bloch, Nature (London) 419, 51 (2002).

[62] T. Kinoshita, T. Wenger, and D. S. Weiss, Nature (London) 440, 900 (2006).

[63] J. Simon, W. S. Bakr, R. Ma, M. E. Tai, P. M. Preiss, and M. Greiner, Nature (London) 472, 307 (2011). 